\title{
Efficient Algorithm for Estimating the Parameters of Two Dimensional Chirp SIGNAL
}

\author{
AnAnya Lahiri $^{1,2} \&$ Debasis Kundu ${ }^{1,3,4}$ \& Amit Mitra ${ }^{1,3}$
}

\begin{abstract}
Two dimensional chirp signal has been used for modeling gray scale symmetric images in the statistical signal processing literature. In this paper we propose a computationally efficient algorithm for estimating different parameters of a two dimensional chirp signal model in presence of stationary noise. Starting from a suitable initial guess value, the proposed method produces estimators which are asymptotically equivalent to the corresponding least squares estimators. We also discuss how to obtain the initial estimates suitably. Some simulation experiments have been performed to see the effectiveness of the proposed method, and it is observed that the proposed estimators perform very well.
\end{abstract}

Key Words and Phrases: Chirp signals; least squares estimators; strong consistency, asymptotic distribution; linear processes; iteration.

${ }^{1}$ Department of Mathematics and Statistics, Indian Institute of Technology Kanpur, Pin 208016, India.

${ }^{2}$ Part of this work has been supported by a grant from the Center for Scientific and Industrial Research (CSIR), Government of India.

${ }^{3}$ Part of this work has been supported by a grant from the Department of Science and Technology, Government of India.

${ }^{4}$ Corresponding author. E-mail: kundu@iitk.ac.in, Fax: 91-512-2597141. 


\section{INTRODUCTION}

Two dimensional chirp signal models can be used to model gray scale (black and white) images. Mathematically the model looks like as follows,

$$
\begin{gathered}
\left.y(m, n)=A^{0} \cos \left(\alpha^{0} m+\beta^{0} m^{2}+\gamma^{0} n+\delta^{0} n^{2}\right)+B^{0} \sin \left(\alpha^{0} m+\beta^{0} m^{2}+\gamma^{0} n+\delta^{0} n^{2}\right)\right] \\
+X(m, n) ; m=1, \cdots, M, n=1, \cdots, N
\end{gathered}
$$

where $X(m, n)$ is the random additive error. $A^{0}$ and $B^{0}$ are distinct non zero amplitudes and $A^{0^{2}}+B^{0^{2}} \leq M$, for some constant $M$. The frequencies $\alpha^{0}, \gamma^{0}$ and frequency rates $\beta^{0}, \delta^{0}$ respectively strictly lie between 0 and $\pi$. It is assumed that $X(m, n)$ has the following form;

$$
X(m, n)=\sum_{j=-\infty}^{\infty} \sum_{k=-\infty}^{\infty} a(j, k) \varepsilon(m-j, n-k)
$$

with

$$
\sum_{j=-\infty}^{\infty} \sum_{k=-\infty}^{\infty}|a(j, k)|<\infty .
$$

Here $\varepsilon(m, n)$ 's are independent and identically distributed (i.i.d.) random variables with zero mean, variance $\sigma^{2}$ and with finite fourth moments. Given a sample of size $M N$, one interesting problem is to estimate the unknown amplitudes $A^{0}, B^{0}$, the frequencies $\alpha^{0}, \gamma^{0}$ and the frequency rates $\beta^{0}, \delta^{0}$.

Extensive work on similar two-dimensional models have been done by several authors, see for example Peleg and Porat [9], Friedlander et al. [6], Francos et al. [4], [5], Cao et al. [2], Zhang and Liu [13], Zhang et al. [14] and the references cited therein. Recently, it is observed by the authors, see Lahiri et al. [8], that the least squares estimators (LSEs) of the parameters of this model (1) are consistent and asymptotically normally distributed. It is further observed that the LSEs are asymptotically efficient. The convergence rates for the amplitudes are $O_{p}\left(M^{-1 / 2} N^{-1 / 2}\right)$, for the frequencies are $O_{p}\left(M^{-3 / 2} N^{-1 / 2}\right)$ and $O_{p}\left(M^{-1 / 2} N^{-3 / 2}\right)$, for the frequency rates these are $O_{p}\left(M^{-5 / 2} N^{-1 / 2}\right)$ and $O_{p}\left(M^{-1 / 2} N^{-5 / 2}\right)$. Here $Z=O_{p}\left(N^{-\delta}\right)$ means $N^{\delta} Z$ is bounded 
in probability. But finding the LSEs is a computationally challenging problem as the least squares surface has several local minima. So for any iterative method the choice of the initial guess is extremely important, otherwise the method will converge to a local minima instead of the global one. Even for ordinary sinusoidal model it is a well known problem, interested readers may see the nice discussion in Rice and Rosenblatt [10] in this respect.

Though we are interested in estimating all the parameters but here in this paper the aim is to find efficient estimators of the amplitudes, frequencies and frequency rates efficiently, which have the same rates of convergence as the corresponding LSEs. It may be observed that the model (1) can be seen as a non-linear regression model, with $A^{0}, B^{0}$ as linear parameters, and $\alpha^{0}, \beta^{0}, \gamma^{0}$ and $\delta^{0}$ as non-linear parameters. If we can find efficient estimators of the non-linear parameters $\alpha^{0}, \beta^{0}, \gamma^{0}$ and $\delta^{0}$ then efficient estimators of the linear parameters can be obtained by simple linear regression technique, see for example Richards [11]. Because of this reason, in this paper we mainly concentrate on estimating the non-linear parameters efficiently.

Here we propose an iterative method (Newton- Raphson type) to find the estimators of non-linear parameters namely frequencies and frequency rates. It is observed that if we start the initial guesses of $\alpha^{0}$ and $\gamma^{0}$ with convergence rates $O_{p}\left(M^{-1} N^{-1 / 2}\right)$ and $O_{p}\left(N^{-1} M^{-1 / 2}\right)$ respectively then after four iterations the algorithm produces the estimates of $\alpha^{0}$ and $\gamma^{0}$ with convergence rates $O_{p}\left(M^{-3 / 2} N^{-1 / 2}\right)$ and $O_{p}\left(M^{-1 / 2} N^{-3 / 2}\right)$. Similarly, if we start the initial guessed of $\beta^{0}$ and $\delta^{0}$ with convergence rates $O_{p}\left(M^{-2} N^{-1 / 2}\right)$ and $O_{p}\left(N^{-2} M^{-1 / 2}\right)$ respectively, then after four iterations the algorithm converges with convergence rates $O_{p}\left(M^{-5 / 2} N^{-1 / 2}\right)$ and $O_{p}\left(M^{-1 / 2} N^{-5 / 2}\right)$. Therefore, it is clear that the proposed algorithm produces estimates which have the same rate of convergence as the LSEs. Moreover, it is known that the algorithm stops after finite number of steps only.

We study the effectiveness of the proposed iterative approach by some simulation 
experiments. It is observed that the algorithm works very well in terms of mean squared errors (MSEs). MSEs of the proposed estimators are very close to the corresponding MSEs of the LSEs, and both are very close to the corresponding asymptotic variance of the LSEs. Therefore, the proposed method can be used very effectively instead of the LSEs.

The rest of the paper is organized as follows. We provide the properties of the LSEs in Section 2. In Section 3, we present the proposed algorithm and provide the theoretical justification of the algorithm. The simulation results and the analysis of a simulated data have been presented in Section 4. Conclusions appear in Section 5. All the proofs are presented in the Appendix.

\section{Least Squares Estimators}

In this section we present briefly the properties of the LSEs for ready reference. The LSEs of the unknown parameters of the model (1) can be obtained by minimizing $S(A, B, \alpha, \beta, \gamma, \delta)$ with respect to $A, B, \alpha, \beta, \gamma$ and $\delta$, where

$$
\begin{aligned}
& S(A, B, \alpha, \beta, \gamma, \delta)= \\
& \quad \sum_{n=1}^{N} \sum_{m=1}^{M}\left(y(m, n)-A \cos \left(\alpha m+\beta m^{2}+\gamma n+\delta n^{2}\right)-B \sin \left(\alpha m+\beta m^{2}+\gamma n+\delta n^{2}\right)\right)^{2} .
\end{aligned}
$$

Note that, if $\alpha, \beta, \gamma$ and $\delta$ are known, the LSEs of $A$ and $B$ can be obtained as $\widehat{A}(\boldsymbol{\theta})$ and $\widehat{B}(\boldsymbol{\theta})$ respectively, where $\boldsymbol{\theta}=(\alpha, \beta, \gamma, \delta)$,

$$
(\widehat{A}(\boldsymbol{\theta}), \widehat{B}(\boldsymbol{\theta}))^{T}=\left(\mathbf{W}^{T}(\boldsymbol{\theta}) \mathbf{W}(\boldsymbol{\theta})\right)^{-1} \mathbf{W}^{T}(\boldsymbol{\theta}) \mathbf{Y}
$$

$\mathbf{Y}=(y(1,1), \cdots, y(M, N))^{T}$, is the $M N \times 1$ data vector and $\mathbf{W}(\boldsymbol{\theta})$ is the $M N \times 2$ matrix of the following form; 


$$
\mathbf{W}(\boldsymbol{\theta})=\left[\begin{array}{cc}
\cos (\alpha+\beta+\gamma+\delta) & \sin (\alpha+\beta+\gamma+\delta) \\
\cos (2 \alpha+4 \beta+\gamma+\delta) & \sin (2 \alpha+4 \beta+\gamma+\delta) \\
\vdots & \vdots \\
\cos \left(M \alpha+M^{2} \beta^{2}+\gamma+\delta\right) & \sin \left(M \alpha+M^{2} \beta^{2}+\gamma+\delta\right) \\
\vdots & \vdots \\
\cos \left(\alpha+\beta+N \gamma+N^{2} \delta\right) & \sin \left(\alpha+\beta+N \gamma+N^{2} \delta\right) \\
\cos \left(2 \alpha+4 \beta+N \gamma+N^{2} \delta\right) & \sin \left(2 \alpha+4 \beta+N \gamma+N^{2} \delta\right) \\
\vdots & \vdots \\
\cos \left(M \alpha+M^{2} \beta^{2}+N \gamma+N^{2} \delta\right) & \sin \left(M \alpha+M^{2} \beta^{2}+N \gamma+N^{2} \delta\right)
\end{array}\right]
$$

Therefore, the LSEs of $\alpha, \beta, \gamma$ and $\delta$ can be obtained first by maximizing $Q(\alpha, \beta, \gamma, \delta)$ with respect to $\alpha, \beta, \gamma$ and $\delta$, where

$$
Q(\alpha, \beta, \gamma, \delta)=S(\widehat{A}(\boldsymbol{\theta}), \widehat{B}(\boldsymbol{\theta}), \alpha, \beta, \gamma, \delta)=\mathbf{Y}^{T} \mathbf{W}(\boldsymbol{\theta})\left(\mathbf{W}^{T}(\boldsymbol{\theta}) \mathbf{W}(\boldsymbol{\theta})\right)^{-1} \mathbf{W}^{T}(\boldsymbol{\theta}) \mathbf{Y}
$$

Once the LSEs of $\alpha, \beta, \gamma$ and $\delta$, say $\widehat{\alpha}, \widehat{\beta}, \widehat{\gamma}$ and $\widehat{\delta}$ are obtained the LSEs of $A$ and $B$ can be easily obtained as $\widehat{A}(\widehat{\alpha}, \widehat{\beta}, \widehat{\gamma}, \widehat{\delta})$ and $\widehat{B}(\widehat{\alpha}, \widehat{\beta}, \widehat{\gamma}, \widehat{\delta})$ respectively, see for example Richards [11]. The properties of the LSEs is as follows. The LSEs of the unknown parameters of model (1) are strongly consistent for the corresponding parameters and they have the following asymptotic distribution

$$
D^{-1}\left(\widehat{A}-A^{0}, \widehat{B}-B^{0}, \widehat{\alpha}-\alpha^{0}, \widehat{\beta}-\beta^{0}, \widehat{\gamma}-\gamma^{0}, \widehat{\delta}-\delta^{0}\right)^{T} \stackrel{d}{\longrightarrow} N_{6}\left(0,2 c \sigma^{2} \Sigma^{-1}\right)
$$

where $D=\operatorname{diag}\left(\frac{1}{\sqrt{M N}}, \frac{1}{\sqrt{M N}}, \frac{1}{M \sqrt{M N}}, \frac{1}{M^{2} \sqrt{M N}}, \frac{1}{N \sqrt{M N}}, \frac{1}{N^{2} \sqrt{M N}}\right)$

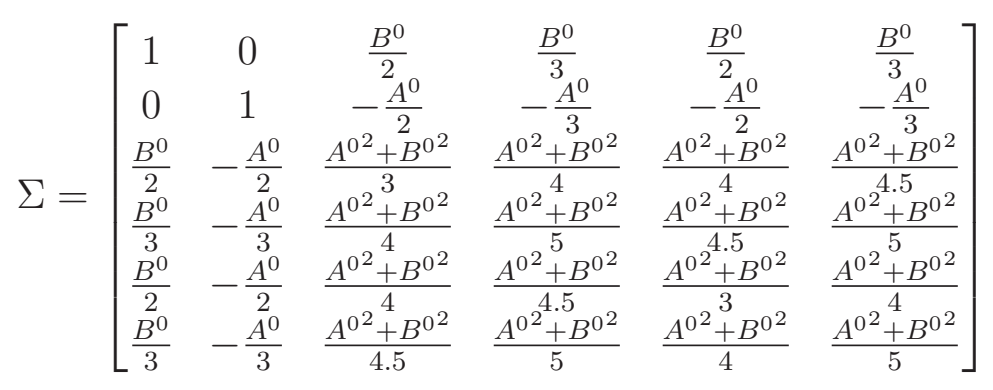

and $c=\sum_{j=-\infty}^{\infty} \sum_{k=-\infty}^{\infty} a(j, k)^{2}$ and

$$
\Sigma^{-1}=\frac{2}{A^{0^{2}}+B^{0^{2}}}\left[\begin{array}{cccccc}
\frac{A^{0^{2}}+17 B^{0^{2}}}{2} & -8 A^{0} B^{0} & -18 B^{0} & 15 B^{0} & -18 B^{0} & 15 B^{0} \\
-8 A^{0} B^{0} & \frac{A^{02}+17 B^{0^{2}}}{2} & 18 A^{0} & -15 A^{0} & 18 A^{0} & -15 A^{0} \\
-18 B^{0} & 18 A^{0} & 96 & -90 & 0 & 0 \\
15 B^{0} & -15 A^{0} & -90 & -90 & 0 & 0 \\
-18 B^{0} & 18 A^{0} & 0 & 0 & 96 & -90 \\
15 B^{0} & -15 A^{0} & 0 & 0 & -90 & 90
\end{array}\right] .
$$


In the next section we provide the algorithm which produces estimators of frequencies $\alpha^{0}, \gamma^{0}$ and frequency rates $\beta^{0}, \delta^{0}$ which have the same asymptotic distributions as the LSEs.

\section{Proposed Algorithm}

The aim of this algorithm is to find the estimates of the frequency and frequency rate with the same rate of convergence as the LSEs. First we will show that starting from any estimators of $\alpha, \beta, \gamma$, and $\delta$, how they can be improved upon. Then we will provide the exact algorithm how it can be implemented in practice. If $\widetilde{\alpha}$ is an estimator of $\alpha^{0}$, such that $\widetilde{\alpha}-\alpha^{0}=O_{p}\left(M^{\left(-1-\lambda_{11}\right)} N^{\left(-\lambda_{12}\right)}\right)$, for some $\frac{1}{2} \geq \lambda_{11}>0$, $\lambda_{12}>0$, and $\widetilde{\beta}$ is an estimator of $\beta^{0}$, such that $\widetilde{\beta}-\beta^{0}=O_{p}\left(M^{\left(-2-\lambda_{21}\right)} N^{\left(-\lambda_{22}\right)}\right)$, for some $\frac{1}{2} \geq \lambda_{21}>0, \lambda_{22}>0$, then an improved estimator of $\alpha^{0}$ can be obtained as

$$
\widetilde{\widetilde{\alpha}}=\widetilde{\alpha}+\frac{48}{M^{2}} \operatorname{Im}\left(\frac{P_{M N}^{\alpha}}{Q_{M N}^{\alpha, \beta}}\right)
$$

with

$$
\begin{gathered}
P_{M N}^{\alpha}=\sum_{n=1}^{N} \sum_{m=1}^{M} y(m, n)\left(m-\frac{M}{2}\right) e^{-i\left(\widetilde{\alpha} m+\widetilde{\beta} m^{2}\right)} \\
Q_{M N}^{\alpha, \beta}=\sum_{n=1}^{N} \sum_{m=1}^{M} y(m, n) e^{-i\left(\widetilde{\alpha} m+\widetilde{\beta} m^{2}\right)} .
\end{gathered}
$$

Here $\operatorname{Im}(\cdot)$ denotes the imaginary part of the complex argument. Similarly, an improved estimator of $\beta^{0}$ can be obtained as

$$
\widetilde{\widetilde{\beta}}=\widetilde{\beta}+\frac{45}{M^{4}} \operatorname{Im}\left(\frac{P_{M N}^{\beta}}{Q_{M N}^{\alpha, \beta}}\right),
$$

with

$$
P_{M N}^{\beta}=\sum_{n=1}^{N} \sum_{m=1}^{M} y(m, n)\left(m^{2}-\frac{M^{2}}{3}\right) e^{-i\left(\widetilde{\alpha} m+\widetilde{\beta} m^{2}\right)}
$$

and $Q_{M N}^{\alpha, \beta}$ is same as defined in (11).

The following two theorems provide the justification for the improved estimators, whose proofs will be provided in the appendix. 
Theorem 1. If $\widetilde{\alpha}-\alpha^{0}=O_{p}\left(M^{\left(-1-\lambda_{11}\right)} N^{\left(-\lambda_{12}\right)}\right)$ for $\lambda_{12}>0$ then

(a) $\widetilde{\widetilde{\alpha}}-\alpha^{0}=O_{p}\left(M^{\left(-1-2 \lambda_{11}\right)} N^{\left(-\lambda_{12}\right)}\right) \quad$ if $\quad 0<\lambda_{11} \leq \frac{1}{4}$

(b) $M^{\frac{3}{2}} N^{\frac{1}{2}}\left(\widetilde{\widetilde{\alpha}}-\alpha^{0}\right) \stackrel{d}{\longrightarrow} \mathrm{N}\left(0, \sigma_{1}^{2}\right) \quad$ if $\quad \lambda_{11}>\frac{1}{4}$,

where $\sigma_{1}^{2}=\frac{384 c \sigma^{2}}{A^{0^{2}}+B^{0^{2}}}$, the asymptotic variance of the LSE of $\alpha^{0}$.

Theorem 2. If $\widetilde{\beta}-\beta^{0}=O_{p}\left(M^{\left(-2-\lambda_{21}\right)} N^{\left(-\lambda_{22}\right)}\right)$ for $\lambda_{22}>0$ then

(a) $\widetilde{\widetilde{\beta}}-\beta^{0}=O_{p}\left(M^{\left(-2-2 \lambda_{21}\right)} N^{\left(-\lambda_{22}\right)}\right) \quad$ if $\quad 0<\lambda_{21} \leq \frac{1}{4}$

(b) $M^{\frac{5}{2}} N^{\frac{1}{2}}\left(\widetilde{\widetilde{\beta}}-\beta^{0}\right) \stackrel{d}{\longrightarrow} \mathrm{N}\left(0, \sigma_{2}^{2}\right) \quad$ if $\quad \lambda_{21}>\frac{1}{4}$

where $\sigma_{2}^{2}=\frac{360 c \sigma^{2}}{A^{0^{2}}+B^{0^{2}}}$, the asymptotic variance of the LSE of $\beta^{0}$.

Similarly by interchanging the role of $m$ and $n$ we can get the estimators of $\gamma^{0}$, and $\delta^{0}$. If $\widetilde{\gamma}$ is an estimator of $\gamma^{0}$, such that $\widetilde{\gamma}-\gamma^{0}=O_{p}\left(N^{\left(-1-\kappa_{11}\right)} M^{\left(-\kappa_{12}\right)}\right)$, for some $\frac{1}{2} \geq \kappa_{11}>0$, and $\widetilde{\delta}$ is an estimator of $\delta^{0}$, such that $\widetilde{\delta}-\delta^{0}=O_{p}\left(N^{\left(-2-\kappa_{21}\right)} M^{\left(-\kappa_{22}\right)}\right)$, for some $\frac{1}{2} \geq \kappa_{21}>0$, then an improved estimator of $\gamma^{0}$ can be obtained as

$$
\widetilde{\widetilde{\gamma}}=\widetilde{\gamma}+\frac{48}{N^{2}} \operatorname{Im}\left(\frac{P_{M N}^{\gamma}}{Q_{M N}^{\gamma, \delta}}\right)
$$

with

$$
\begin{gathered}
P_{M N}^{\gamma}=\sum_{n=1}^{N} \sum_{m=1}^{M} y(m, n)\left(n-\frac{N}{2}\right) e^{-i\left(\widetilde{\gamma} n+\widetilde{\delta} n^{2}\right)} \\
Q_{M N}^{\gamma, \delta}=\sum_{n=1}^{N} \sum_{m=1}^{M} y(m, n) e^{\left(-i \widetilde{\gamma} n+\widetilde{\delta} n^{2}\right)} .
\end{gathered}
$$

and an improved estimator of $\delta^{0}$ can be obtained as

$$
\widetilde{\widetilde{\delta}}=\widetilde{\delta}+\frac{45}{N^{4}} \operatorname{Im}\left(\frac{P_{M N}^{\delta}}{Q_{M N}^{\gamma, \delta}}\right),
$$

with

$$
P_{M N}^{\delta}=\sum_{n=1}^{N} \sum_{m=1}^{M} y(m, n)\left(n^{2}-\frac{N^{2}}{3}\right) e^{-i\left(\widetilde{\gamma} n+\widetilde{\delta} n^{2}\right)}
$$

and $Q_{M N}^{\gamma, \delta}$ is same as defined in (16).

Theorem 3. If $\widetilde{\gamma}-\gamma^{0}=O_{p}\left(N^{\left(-1-\kappa_{11}\right)} M^{\left(-\kappa_{12}\right)}\right)$ for $\kappa_{12}>0$ then
(a) $\widetilde{\widetilde{\gamma}}-\gamma^{0}=O_{p}\left(N^{\left(-1-2 \kappa_{11}\right)} M^{\left(-\kappa_{12}\right)}\right)$
if $\quad 0<\kappa_{11} \leq \frac{1}{4}$
(b) $N^{\frac{3}{2}} M^{\frac{1}{2}}\left(\widetilde{\widetilde{\gamma}}-\gamma^{0}\right) \stackrel{d}{\longrightarrow} \mathrm{N}\left(0, \sigma_{1}^{2}\right) \quad$ if $\quad \kappa_{11}>\frac{1}{4}$, 
Theorem 4. If $\widetilde{\delta}-\delta^{0}=O_{p}\left(N^{\left(-2-\kappa_{21}\right)} M^{\left(-\kappa_{22}\right)}\right)$ for $\kappa_{22}>0$ then

(a) $\widetilde{\widetilde{\delta}}-\delta^{0}=O_{p}\left(N^{\left(-2-2 \kappa_{21}\right)} M^{\left(-\kappa_{22}\right)}\right) \quad$ if $\quad 0<\kappa_{21} \leq \frac{1}{4}$

(b) $N^{\frac{5}{2}} M^{\frac{1}{2}}\left(\widetilde{\widetilde{\delta}}-\delta^{0}\right) \stackrel{d}{\longrightarrow} \mathrm{N}\left(0, \sigma_{2}^{2}\right) \quad$ if $\quad \kappa_{21}>\frac{1}{4}$.

Now we will show that starting from initial guesses $\widetilde{\alpha}, \widetilde{\beta}$, with convergence rates $\widetilde{\alpha}-\alpha^{0}=O_{p}\left(M^{-1} N^{-1 / 2}\right)$ and $\widetilde{\beta}-\beta^{0}=O_{p}\left(M^{-2} N^{-1 / 2}\right)$ respectively, how the above procedure can be used to obtain efficient estimators. It may be noted that finding initial guesses with the above convergence rates are not very difficult. It can be obtained by finding the minimum of $Q_{1}(\alpha, \beta)$, where

$$
Q_{1}(\alpha, \beta)=\sum_{m=1}^{M}\left(\sum_{n=1}^{N} y(m, n)-\mathcal{A} \cos \left(\alpha m+\beta m^{2}\right)-\mathcal{B} \sin \left(\alpha m+\beta m^{2}\right)\right)^{2}
$$

over the grids $\left(\frac{\pi j}{M}, \frac{\pi k}{M^{2}}\right), j=1, \cdots, M$, and $k=1, \cdots, M^{2}$, as it has been suggested by Rice and Rosenblatt [10] to find the initial guesses of the frequency in case of a sinusoidal model.

The main idea is not to use the whole sample size at the beginning, as it was first suggested by Bai et al. [1]. We will use part of the sample at the beginning and gradually proceed towards the complete sample. The algorithm can be described as follows. We will denote the estimates of $\alpha^{0}$ and $\beta^{0}$ obtained at the $i$-th iteration as $\widetilde{\alpha}^{(i)}$ and $\widetilde{\beta}^{(i)}$ respectively.

\section{Algorithm:}

Step 1: Choose $M_{1}=M^{8 / 9}, N_{1}=N$. Therefore, $\widetilde{\alpha}^{(0)}-\alpha^{0}=O_{p}\left(M^{-1} N^{-1 / 2}\right)=$ $O_{p}\left(M_{1}^{-1-1 / 8} N_{1}^{-1 / 2}\right)$, and $\widetilde{\beta}^{(0)}-\beta^{0}=O_{p}\left(M^{-2} N^{-1 / 2}\right)=O_{p}\left(M_{1}^{-2-1 / 4} N_{1}^{-1 / 2}\right)$. Perform steps (9) and (12). Therefore, after the 1-st iteration we have

$$
\widetilde{\alpha}^{(1)}-\alpha^{0}=O_{p}\left(M_{1}^{-1-1 / 4} N_{1}^{-1 / 2}\right)=O_{p}\left(M^{-10 / 9} N^{-1 / 2}\right)
$$

and

$$
\widetilde{\beta}^{(1)}-\beta^{0}=O_{p}\left(M_{1}^{-2-\frac{1}{2}} N_{1}^{-1 / 2}\right)=O_{p}\left(M^{-20 / 9} N^{-1 / 2}\right) .
$$


Step 2: Choose $M_{2}=M^{80 / 81}, N_{2}=N$. Therefore, $\widetilde{\alpha}^{(1)}-\alpha^{0}=O_{p}\left(M_{2}^{-1-1 / 8} N_{2}^{-1 / 2}\right)$, and $\quad \widetilde{\beta}^{(1)}-\beta^{0}=O_{p}\left(M_{2}^{-2-1 / 4} N_{2}^{-1 / 2}\right)$. Perform steps (9) and (12). Therefore, after the 2-nd iteration we have

$$
\widetilde{\alpha}^{(2)}-\alpha^{0}=O_{p}\left(M_{2}^{-1-1 / 4} N_{2}^{-1 / 2}\right)=O_{p}\left(M^{-100 / 81} N^{-1 / 2}\right)
$$

and

$$
\widetilde{\beta}^{(2)}-\beta^{0}=O_{p}\left(M_{2}^{-2-1 / 2} N_{2}^{-1 / 2}\right)=O_{p}\left(M^{-200 / 81} N^{-1 / 2}\right) .
$$

Step 3: Choose $M_{3}=M, N_{3}=N$. Therefore, $\widetilde{\alpha}^{(2)}-\alpha^{0}=O_{p}\left(M_{3}^{-1-19 / 81} N_{3}^{-1 / 2}\right)$, and $\quad \widetilde{\beta}^{(2)}-\beta^{0}=O_{p}\left(M_{3}^{-2-38 / 81} N_{3}^{-1 / 2}\right)$. Perform steps (9) and (12). Therefore, after the 3-rd iteration we have

$$
\widetilde{\alpha}^{(3)}-\alpha^{0}=O_{p}\left(M^{-1-38 / 81} N^{-1 / 2}\right), \quad \text { and } \quad \widetilde{\beta}^{(3)}-\beta^{0}=O_{p}\left(M^{-2-76 / 81} N^{-1 / 2}\right) .
$$

Step 4: Choose $M_{4}=M, N_{4}=N$, and perform steps (9) and (12). Now we obtain the required convergence rates, i.e.

$$
\widetilde{\alpha}^{(4)}-\alpha^{0}=O_{p}\left(M^{-3 / 2} N^{-1 / 2}\right), \quad \text { and } \quad \widetilde{\beta}^{(4)}-\beta^{0}=O_{p}\left(M^{-5 / 2} N^{-1 / 2}\right) .
$$

Similarly interchanging the role of $m$ and $n$ we can get the algorithm corresponding to $\gamma^{0}$ and $\delta^{0}$.

\section{Simulation AND DATA ANALYSis}

\subsection{Simulation Results}

In this section we present some simulation results for different sample sizes and for different error variances to show how the proposed method behaves in practice. We consider the following model

$\left.y(m, n)=5.0 \cos \left(1.0 m+0.05 m^{2}+1.5 n+0.5 n^{2}\right)+5.0 \sin \left(1.0 m+0.05 m^{2}+1.5 n+0.5 n^{2}\right)\right)$. 
Table 1: Comparison of LSE and Algorithm for 2D chirp model with i.i.d. error

\begin{tabular}{|c|c|c|c|c|c|}
\hline \multicolumn{2}{|c|}{} & \multicolumn{4}{|c|}{ sample size $=50 \times 50$} \\
\hline$\sigma^{2}=0.05$ & PARA & $\alpha^{0}=1.00$ & $\beta^{0}=0.05$ & $\gamma^{0}=1.50$ & $\delta^{0}=0.50$ \\
& ASYV & $(0.6144001 \mathrm{E}-07)$ & $(0.2304000 \mathrm{E}-10)$ & $(0.6144001 \mathrm{E}-07)$ & $(0.2304000 \mathrm{E}-10)$ \\
& MEAN(Algo) & 1.000093 & 0.049997 & 1.500015 & 0.500000 \\
& MSE(Algo) & $(0.5010137 \mathrm{E}-04)$ & $(0.2194539 \mathrm{E}-07)$ & $(0.2086442 \mathrm{E}-04)$ & $(0.7875175 \mathrm{E}-08)$ \\
& MEAN(LSE) & 0.999997 & 0.050000 & 1.500020 & 0.500000 \\
& MSE(LSE) & $(0.1124367 \mathrm{E}-06)$ & $(0.3985947 \mathrm{E}-10)$ & $(0.3852298 \mathrm{E}-06)$ & $(0.1130815 \mathrm{E}-09)$ \\
\hline$\sigma^{2}=0.5$ & PARA & $\alpha^{0}=1.00$ & $\beta^{0}=0.05$ & $\gamma^{0}=1.50$ & $\delta^{0}=0.50$ \\
& ASYV & $(0.6144001 \mathrm{E}-06)$ & $(0.2304000 \mathrm{E}-09)$ & $(0.6144001 \mathrm{E}-06)$ & $(0.2304000 \mathrm{E}-09)$ \\
& MEAN(Algo) & 1.000108 & 0.049992 & 1.499921 & 0.500004 \\
& MSE(Algo) & $(0.8032256 \mathrm{E}-04)$ & $(0.5519645 \mathrm{E}-07)$ & $(0.6177288 \mathrm{E}-04)$ & $(0.2731907 \mathrm{E}-07)$ \\
& MEAN(LSE) & 1.000072 & 0.049999 & 1.500018 & 0.499999 \\
& MSE(LSE) & $(0.1133311 \mathrm{E}-05)$ & $(0.4220677 \mathrm{E}-09)$ & $(0.5684647 \mathrm{E}-05)$ & $(0.2187304 \mathrm{E}-08)$ \\
\hline
\end{tabular}

Table 2: Comparison of LSE and Algorithm for 2D chirp model with i.i.d. error

\begin{tabular}{|c|c|c|c|c|c|}
\hline \multicolumn{2}{|c|}{} & \multicolumn{4}{|c|}{ sample size $=75 \times 75$} \\
\hline$\sigma^{2}=0.05$ & PARA & $\alpha^{0}=1.00$ & $\beta^{0}=0.05$ & $\gamma^{0}=1.50$ & $\delta^{0}=0.50$ \\
& ASYV & $(0.1213630 \mathrm{E}-07)$ & $(0.2022716 \mathrm{E}-11)$ & $(0.1213630 \mathrm{E}-07)$ & $(0.2022716 \mathrm{E}-11)$ \\
& MEAN(Algo) & 0.999798 & 0.050002 & 1.500087 & 0.499999 \\
& MSE(Algo) & $(0.1951499 \mathrm{E}-04)$ & $(0.3237120 \mathrm{E}-08)$ & $(0.1740811 \mathrm{E}-05)$ & $(0.2798037 \mathrm{E}-09)$ \\
& MEAN(LSE) & 1.000027 & 0.050000 & 1.499981 & 0.500000 \\
& MSE(LSE) & $(0.3739163 \mathrm{E}-07)$ & $(0.6373457 \mathrm{E}-11)$ & $(0.3754973 \mathrm{E}-07)$ & $(0.6375678 \mathrm{E}-11)$ \\
\hline$\sigma^{2}=0.5$ & PARA & $\alpha^{0}=1.00$ & $\beta^{0}=0.05$ & $\gamma^{0}=1.50$ & $\delta^{0}=0.50$ \\
& ASYV & $(0.1213630 \mathrm{E}-06)$ & $(0.2022716 \mathrm{E}-10)$ & $(0.1213630 \mathrm{E}-06)$ & $(0.2022716 \mathrm{E}-10)$ \\
& MEAN(Algo) & 0.999679 & 0.050003 & 1.500144 & 0.499998 \\
& MSE(Algo) & $(0.5891653 \mathrm{E}-04)$ & $(0.1142206 \mathrm{E}-07)$ & $(0.1408832 \mathrm{E}-04)$ & $(0.2284886 \mathrm{E}-08)$ \\
& MEAN(LSE) & 1.000020 & 0.050000 & 1.500002 & 0.500000 \\
& MSE(LSE) & $(0.2757161 \mathrm{E}-06)$ & $(0.4616362 \mathrm{E}-10)$ & $(0.2735573 \mathrm{E}-06)$ & $(0.4724625 \mathrm{E}-10)$ \\
\hline
\end{tabular}

Table 3: Comparison of LSE and Algorithm for 2D chirp model with stationary error

\begin{tabular}{|c|c|c|c|c|c|}
\hline \multicolumn{1}{|c|}{} & \multicolumn{4}{|c|}{ sample size $=50 \times 50$} \\
\hline$\sigma^{2}=0.05$ & PARA & $\alpha^{0}=1.00$ & $\beta^{0}=0.05$ & $\gamma^{0}=1.50$ & $\delta^{0}=0.50$ \\
& ASYV & $(0.8349082 \mathrm{E}-07)$ & $(0.3130906 \mathrm{E}-10)$ & $(0.8349082 \mathrm{E}-07)$ & $(0.3130906 \mathrm{E}-10)$ \\
& MEAN(Algo) & 1.000111 & 0.049998 & 1.500039 & 0.499999 \\
& MSE(Algo) & $(0.6044609 \mathrm{E}-04)$ & $(0.3092874 \mathrm{E}-07)$ & $(0.3720176 \mathrm{E}-04)$ & $(0.1503450 \mathrm{E}-07)$ \\
& MEAN(LSE) & 1.000016 & 0.050000 & 1.500002 & 0.500000 \\
& MSE(LSE) & $(0.1159275 \mathrm{E}-06)$ & $(0.4430854 \mathrm{E}-10)$ & $(0.1125116 \mathrm{E}-06)$ & $(0.4000090 \mathrm{E}-10)$ \\
\hline$\sigma^{2}=0.5$ & PARA & $\alpha^{0}=1.00$ & $\beta^{0}=0.05$ & $\gamma^{0}=1.50$ & $\delta^{0}=0.50$ \\
& ASYV & $(0.8349081 \mathrm{E}-06)$ & $(0.3130906 \mathrm{E}-09)$ & $(0.8349081 \mathrm{E}-06)$ & $(0.3130906 \mathrm{E}-09)$ \\
& MEAN(Algo) & 1.000086 & 0.050000 & 1.499968 & 0.500003 \\
& MSE(Algo) & $(0.8371484 \mathrm{E}-04)$ & $(0.9488036 \mathrm{E}-07)$ & $(0.7387891 \mathrm{E}-04)$ & $(0.4147575 \mathrm{E}-07)$ \\
& MEAN(LSE) & 1.000068 & 0.049999 & 1.499953 & 0.500001 \\
& MSE(LSE) & $(0.1414497 \mathrm{E}-05)$ & $(0.5333091 \mathrm{E}-09)$ & $(0.1192405 \mathrm{E}-05)$ & $(0.4391810 \mathrm{E}-09)$ \\
\hline
\end{tabular}


Table 4: Comparison of LSE and Algorithm for 2D chirp model with stationary error

\begin{tabular}{|c|c|c|c|c|c|}
\hline \multicolumn{2}{|c|}{} & \multicolumn{4}{|c|}{ sample size $=75 \times 75$} \\
\hline$\sigma^{2}=0.05$ & PARA & $\alpha^{0}=1.00$ & $\beta^{0}=0.05$ & $\gamma^{0}=1.50$ & $\delta^{0}=0.50$ \\
& ASYV & $(0.1649201 \mathrm{E}-07)$ & $(0.2748669 \mathrm{E}-11)$ & $(0.1649201 \mathrm{E}-07)$ & $(0.2748669 \mathrm{E}-11)$ \\
& MEAN(Algo) & 0.999800 & 0.050002 & 1.500034 & 0.500000 \\
& MSE(Algo) & $(0.3097944 \mathrm{E}-04)$ & $(0.4884151 \mathrm{E}-08)$ & $(0.3867856 \mathrm{E}-05)$ & $(0.6546732 \mathrm{E}-09)$ \\
& MEAN(LSE) & 1.000024 & 0.050000 & 1.499987 & 0.500000 \\
& MSE(LSE) & $(0.4411412 \mathrm{E}-07)$ & $(0.7600867 \mathrm{E}-11)$ & $(0.4548108 \mathrm{E}-07)$ & $(0.7872438 \mathrm{E}-11)$ \\
\hline$\sigma^{2}=0.5$ & PARA & $\alpha^{0}=1.00$ & $\beta^{0}=0.05$ & $\gamma^{0}=1.50$ & $\delta^{0}=0.50$ \\
& ASYV & $(0.1649201 \mathrm{E}-06)$ & $(0.2748669 \mathrm{E}-10)$ & $(0.1649201 \mathrm{E}-06)$ & $(0.2748669 \mathrm{E}-10)$ \\
& MEAN(Algo) & 0.999669 & 0.050004 & 1.500081 & 0.499999 \\
& MSE(Algo) & $(0.6820307 \mathrm{E}-04)$ & $(0.1362305 \mathrm{E}-07)$ & $(0.2642028 \mathrm{E}-04)$ & $(0.4555038 \mathrm{E}-08)$ \\
& MEAN(LSE) & 1.000021 & 0.050000 & 1.499990 & 0.500000 \\
& MSE(LSE) & $(0.3979654 \mathrm{E}-06)$ & $(0.6132625 \mathrm{E}-10)$ & $(0.3148316 \mathrm{E}-06)$ & $(0.5325718 \mathrm{E}-10)$ \\
\hline
\end{tabular}

We have considered (i) $X(m, n)=\varepsilon(m, n)$ (i.i.d. errors) and (ii) $X(m, n)=\epsilon(m, n)+$ $0.5 \epsilon(m-1, n)+0.33 \epsilon(m, n-1)$ (stationary error), where $\varepsilon(m, n)$ 's are i.i.d. normal random variables with mean 0 and variance $\sigma^{2}$. We have taken $M=N=50,75$, $\sigma^{2}=0.05$ and 0.5 . In each case we have obtained the initial guesses as has been suggested in Section 3. For each $M=N$ and $\sigma^{2}$, we compute the average estimates of $\alpha^{0}, \beta^{0}, \gamma^{0}$ and $\delta^{0}$ and the associated MSEs based on 1000 replications. The results are reported in Tables 1 to 4 . For comparison purposes we have also computed the LSEs and the corresponding asymptotic variances.

From the results presented in the tables, it is clear that the performances of the estimators obtained by the proposed algorithm are quite satisfactory in comparison to the corresponding performances of the LSEs. The MSEs of the proposed estimators are very close to the asymptotic variance of the corresponding LSEs. The performances are quite good even with moderate sample sizes. It is clear that even with the four steps of iteration, we are able to achieve the same accuracy as of the LSEs.

\subsection{Data Analysis}

In this subsection we present the analysis of a simulated data set mainly for illustrative purposes. We have generated a sample of size $75 \times 75$ from the chirp model with following parameters, $A^{0}=5.0, \quad B^{0}=1.0, \quad \alpha^{0}=1.55, \quad \beta^{0}=0.05, \quad \gamma^{0}=1.25, \quad \delta^{0}=$ 
0.075. $X(n) \mathrm{s}$ are as follows, $X(m, n)=\varepsilon(m, n)+0.5 \varepsilon(m-1, n)+0.33 \varepsilon(m, n-1)$ where $\varepsilon(m, n)$ 's are assumed to be i.i.d. Gaussian random variables with mean 0 and variance $\sigma^{2}=0.5$. We have generated the data $y(m, n)$ for $M=N=75$. We get the initial guess from the least squares surfaces of the row sum and column sum of the data. We also present the two least squares surfaces in Figure 1 and 2 for column sum and row sum of the data respectively.

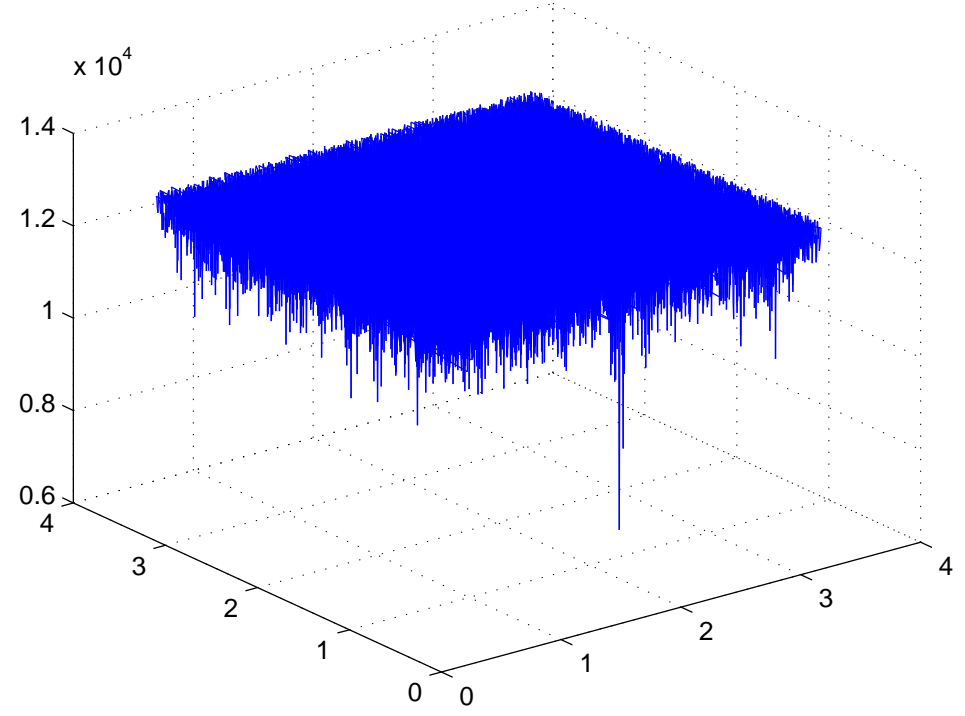

Figure 1: Least squares surface for column sum

Using the algorithm, we obtained the estimates of $A^{0}, B^{0}, \alpha^{0}, \beta^{0}, \gamma^{0}$ and $\delta^{0}$ as $4.996596,0.999698,1.550000,0.050000,1.250000,0.075000$ respectively. We plot the true image, noisy image and estimated image in Figure 3, 4 and 5 respectively. We can see that the original and the estimated plots match quite well.

\section{COnClusion}

In this paper we propose an efficient algorithm to find the estimators of the parameters of a two dimensional chirp signal model which are asymptotically equivalent to LSEs of the corresponding model parameters. To implement the algorithm we 


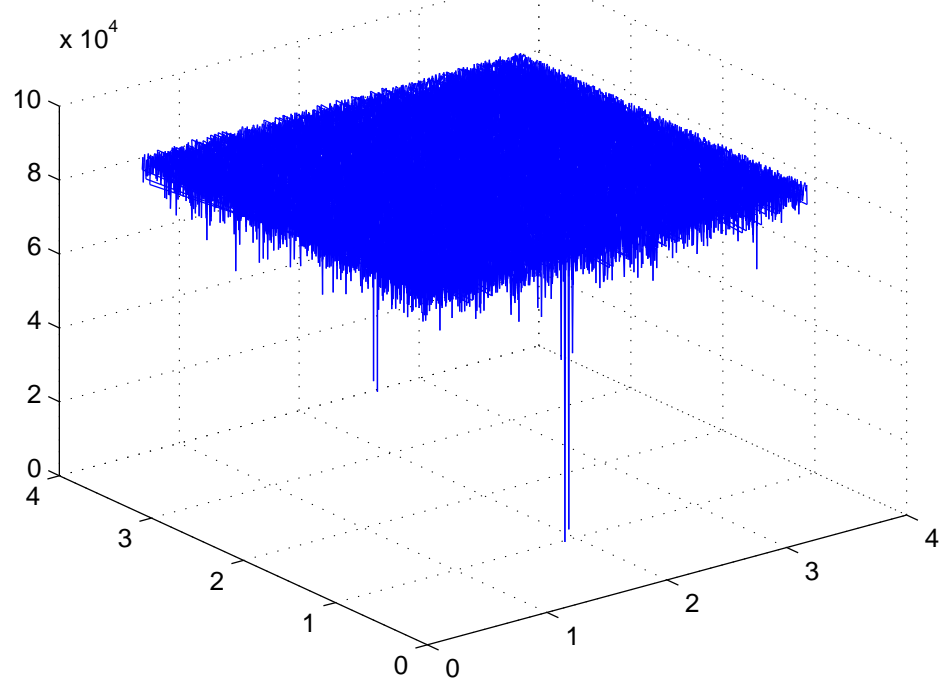

Figure 2: Least square surface for row sum

need a good initial estimate. We have discussed how to get such an initial guess in practice. Simulation results indicate that the proposed method works quite well in terms of MSEs, and it produces estimators which perform like the LSEs. Since the proposed algorithm converges in four steps only, it can be used very effectively for online implementation purposes.

\section{ACKNOWLEDGEMENT}

The authors would like to thank the referees for their very constructive comments, which had helped to improve the manuscript significantly.

\section{APPENDIX}

We need the following lemmas for proving the theorems. Using Vinogradov's [12] result one can prove Lemma 1 and Lemma 2. 


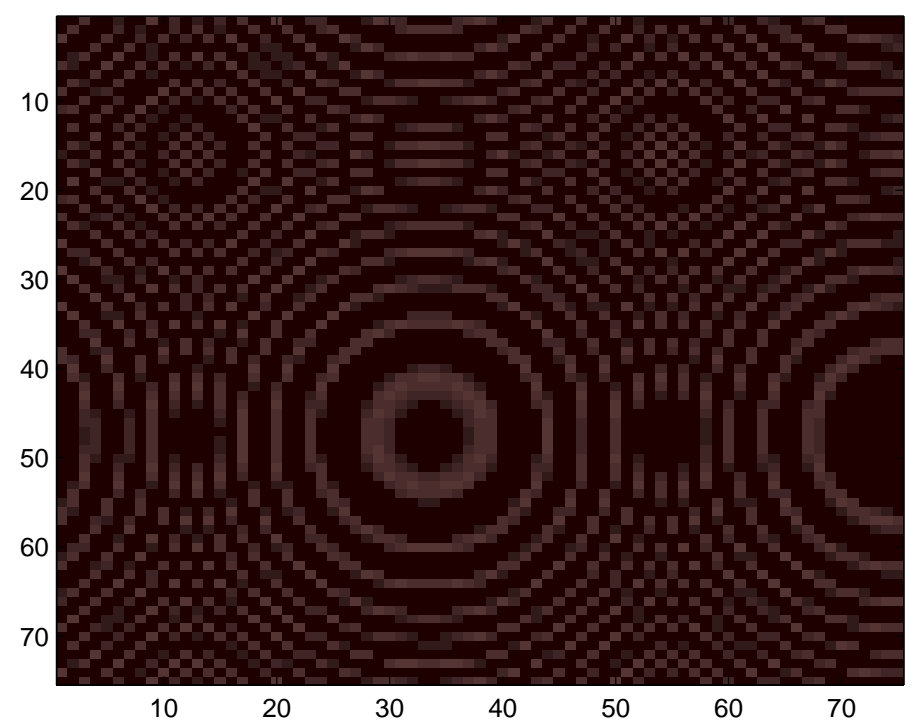

Figure 3: Original texture

Lemma 1. If $(\omega, \theta) \in(0, \pi) \times(0, \pi)$, then except for countable number of points $\lim _{\min \{M, N\} \rightarrow \infty} \frac{1}{M N} \sum_{n=1}^{N} \sum_{m=1}^{M} \cos \left(\omega m^{2}+\theta n^{2}\right)=\lim _{\min \{M, N\} \rightarrow \infty} \frac{1}{M N} \sum_{n=1}^{N} \sum_{m=1}^{M} \sin \left(\omega m^{2}+\theta n^{2}\right)=0$. $\lim _{\min \{M, N\} \rightarrow \infty} \frac{1}{M N} \sum_{n=1}^{N} \sum_{m=1}^{M} \cos ^{2}\left(\omega m^{2}+\theta n^{2}\right)=\lim _{\min \{M, N\} \rightarrow \infty} \frac{1}{M N} \sum_{n=1}^{N} \sum_{m=1}^{M} \sin ^{2}\left(\omega m^{2}+\theta n^{2}\right)=\frac{1}{2}$.

Lemma 2. If $\left(\omega_{1}, \omega_{2}, \theta_{1}, \theta_{2}\right) \in(0, \pi) \times(0, \pi) \times(0, \pi) \times(0, \pi)$, then except for countable number of points

$$
\begin{gathered}
\lim _{\min \{M, N\} \rightarrow \infty} \frac{1}{M N} \sum_{n=1}^{N} \sum_{m=1}^{M} \cos \left(\omega_{1} m+\omega_{2} m^{2}+\theta_{1} n+\theta_{2} n^{2}\right)= \\
\lim _{\min \{M, N\} \rightarrow \infty} \frac{1}{M N} \sum_{n=1}^{N} \sum_{m=1}^{M} \sin \left(\omega_{1} m+\omega_{2} m^{2}+\theta_{1} n+\theta_{2} n^{2}\right)=0 . \\
\lim _{\min \{M, N\} \rightarrow \infty} \frac{1}{M^{(s+1)} N^{(t+1)}} \sum_{n=1}^{N} \sum_{m=1}^{M} m^{s} n^{t} \cos ^{2}\left(\omega_{1} m+\omega_{2} m^{2}+\theta_{1} n+\theta_{2} n^{2}\right)= \\
\lim _{\min \{M, N\} \rightarrow \infty} \frac{1}{M^{(s+1)} N^{(t+1)}} \sum_{n=1}^{N} \sum_{m=1}^{M} m^{s} n^{t} \sin ^{2}\left(\omega_{1} m+\omega_{2} m^{2}+\theta_{1} n+\theta_{2} n^{2}\right)= \\
\frac{1}{2(s+1)(t+1)} .
\end{gathered}
$$




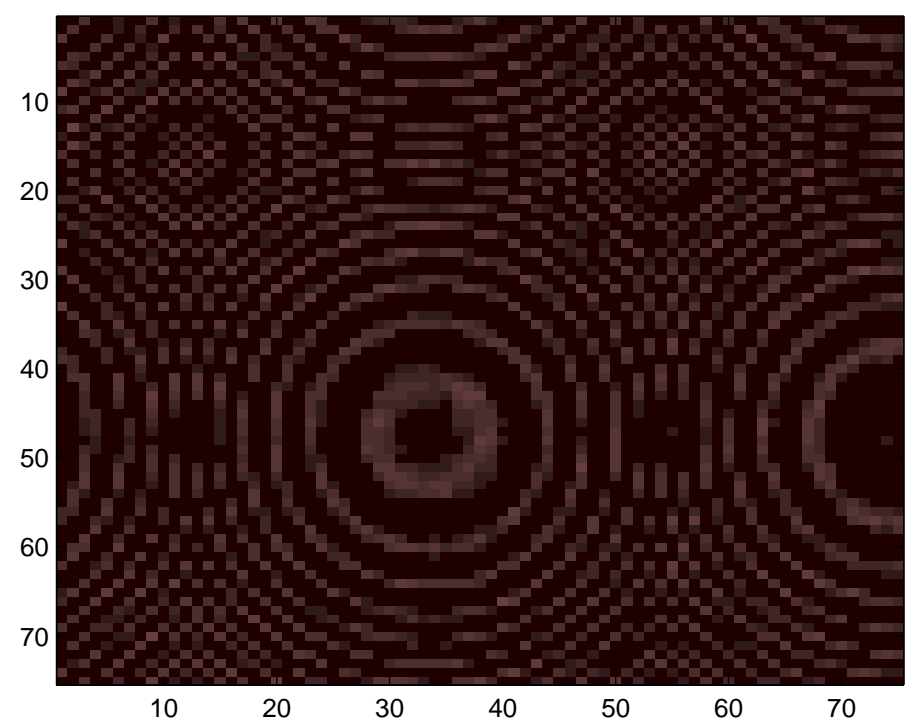

Figure 4: Noisy texture

Lemma 3. $Q_{M N}^{\alpha, \beta}=\left(\frac{A^{0}+B^{0}}{2}+i \frac{A^{0}-B^{0}}{2}\right) O_{p}(M N)$

ProOF

$$
\begin{gathered}
Q_{M N}^{\alpha, \beta}=\sum_{m=1}^{M} \sum_{n=1}^{N} y(m, n) e^{-i\left(\widetilde{\alpha} m+\widetilde{\beta} m^{2}\right)} \\
=\sum_{m=1}^{M}\left(\frac{A_{N}^{*}-i B_{N}^{*}}{2}\right) e^{i\left[\left(\alpha^{0}-\widetilde{\alpha}\right) m+\left(\beta^{0}-\widetilde{\beta}\right) m^{2}\right]}+\sum_{m=1}^{M}\left(\frac{A_{N}^{*}+i B_{N}^{*}}{2}\right) e^{-i\left[\left(\alpha^{0}+\widetilde{\alpha}\right) m+\left(\beta^{0}+\widetilde{\beta}\right) m^{2}\right]} \\
+\sum_{m=1}^{M} \sum_{n=1}^{N} X(m, n) e^{-i\left(\widetilde{\alpha} m+\widetilde{\beta} m^{2}\right)}
\end{gathered}
$$

To get 2nd term in equation (25) we use the Lemma 2 and get

$$
\sum_{m=1}^{M} e^{-i\left[\left(\alpha^{0}+\widetilde{\alpha}\right) m+\left(\beta^{0}+\widetilde{\beta}\right) m^{2}\right]}=o_{p}(M) .
$$

Using Lemma 2 and bivariate Taylor series expansion the 1st term in equation (25) becomes 


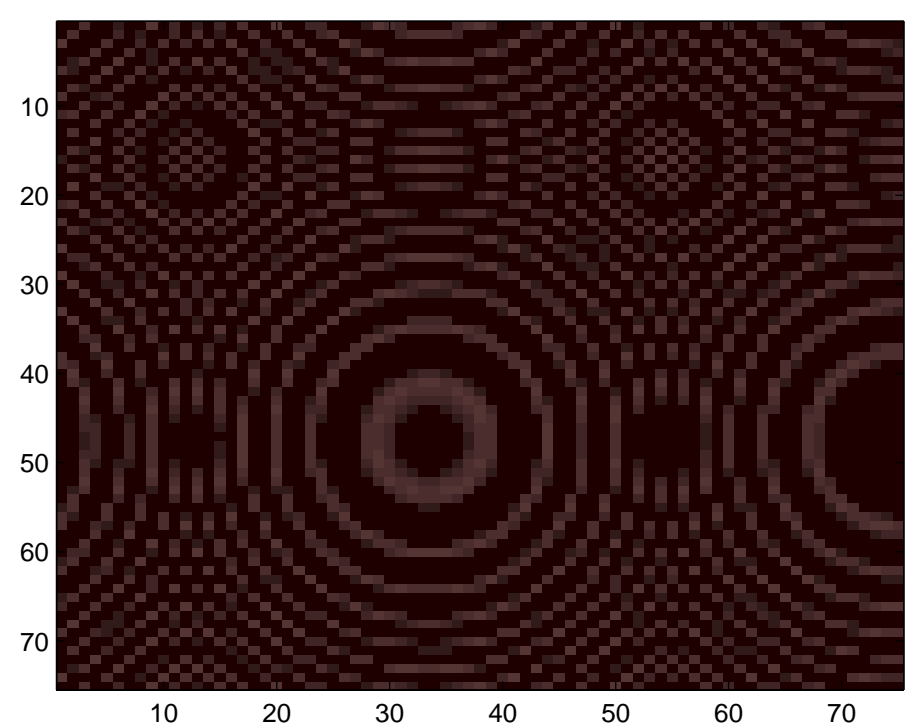

Figure 5: Estimated texture

$$
\begin{aligned}
& \sum_{m=1}^{M} e^{i\left[\left(\alpha^{0}-\widetilde{\alpha}\right) m+\left(\beta^{0}-\widetilde{\beta}\right) m^{2}\right]}=\sum_{m=1}^{M}\left[e^{i\left[m\left(\alpha^{0}-\alpha^{0}\right)+m^{2}\left(\beta^{0}-\beta^{0}\right)\right]}+i\left(\alpha^{0}-\widetilde{\alpha}\right) m+i\left(\beta^{0}-\widetilde{\beta}\right) m^{2}\right. \\
& +\frac{i^{2}\left(\alpha^{0}-\widetilde{\alpha}\right)^{2} m^{2}}{2 !} e^{i\left[m\left(\alpha^{0}-\alpha^{*}\right)+m^{2}\left(\beta^{0}-\beta^{*}\right)\right]}+\frac{i^{2}\left(\beta^{0}-\widetilde{\beta}\right)^{2} m^{4}}{2 !} e^{i\left[m\left(\alpha^{0}-\alpha^{*}\right)+m^{2}\left(\beta^{0}-\beta^{*}\right)\right]} \\
& \left.+\frac{2 i^{2}\left(\alpha^{0}-\widetilde{\alpha}\right)\left(\beta^{0}-\widetilde{\beta}\right) m^{3}}{2 !} e^{i\left[m\left(\alpha^{0}-\alpha^{*}\right)+m^{2}\left(\beta^{0}-\beta^{*}\right)\right]}\right] \\
& =O(M)+O_{p}\left(M^{-1-\lambda_{11}} N^{-\lambda_{12}}\right) O\left(M^{2}\right)+O_{p}\left(M^{-2-\lambda_{21}} N^{-\lambda_{22}}\right) O\left(M^{3}\right) \\
& +\frac{1}{2} O_{p}\left(M^{-2-2 \lambda_{11}} N^{-2 \lambda_{12}}\right) O_{p}\left(M^{3}\right)+\frac{1}{2} O_{p}\left(M^{-4-2 \lambda_{21}} N^{-2 \lambda_{22}}\right) O_{p}\left(M^{5}\right) \\
& +O_{p}\left(M^{-1-\lambda_{11}} N^{-\lambda_{12}}\right) O_{p}\left(M^{-2-\lambda_{21}} N^{-\lambda_{22}}\right) O_{p}\left(M^{4}\right) \\
& =O_{p}(M)+O_{p}\left(M^{1-\lambda_{11}} N^{-\lambda_{12}}\right)+O_{p}\left(M^{1-\lambda_{21}} N^{-\lambda_{22}}\right) \\
& +\frac{1}{2} O_{p}\left(M^{1-2 \lambda_{11}} N^{-2 \lambda_{12}}\right)+O_{p}\left(M^{1-\lambda_{11}-\lambda_{21}} N^{-\lambda_{12}-\lambda_{22}}\right)+\frac{1}{2} O_{p}\left(M^{1-2 \lambda_{21}} N^{-2 \lambda_{22}}\right) \\
& =O_{p}(M)+O_{p}\left(M^{1-\min \left(\lambda_{11}, \lambda_{21}\right)} N^{-\min \left(\lambda_{12}, \lambda_{22}\right)}\right)+O_{p}\left(M^{1-2 \min \left(\lambda_{11}, \lambda_{21}\right)} N^{-2 \min \left(\lambda_{12}, \lambda_{22}\right)}\right) \\
& =O_{p}(M)\left(1+O_{p}\left(M^{-\min \left(\lambda_{11}, \lambda_{21}\right)} N^{-\min \left(\lambda_{12}, \lambda_{22}\right)}\right)+O_{p}\left(M^{-2 \min \left(\lambda_{11}, \lambda_{21}\right)} N^{-2 \min \left(\lambda_{12}, \lambda_{22}\right)}\right)\right)
\end{aligned}
$$

where $\left(\alpha^{*}, \beta^{*}\right)$ is a point on the line joining $\left(\alpha^{0}, \beta^{0}\right)$ and $(\widetilde{\alpha}, \widetilde{\beta})$.

To calculate the 3rd term in equation (25) we need the following two observations. 
(i)

$$
\frac{1}{M^{s+\frac{1}{2}} N^{\frac{1}{2}}} \sum_{m=1}^{M} \sum_{n=1}^{N} m^{s}\left[\sin \left(\alpha^{0} m+\beta^{0} m^{2}\right)\right] X(m, n)
$$

and

$$
\frac{1}{M^{s+\frac{1}{2}} N^{\frac{1}{2}}} \sum_{m=1}^{M} \sum_{n=1}^{N} m^{s}\left[\cos \left(\alpha^{0} m+\beta^{0} m^{2}\right)\right] X(m, n)
$$

satisfy conditions for CLT, see Fuller [3], when $\alpha^{0}, \beta^{0}$ are the true value as in the model.

(ii) $\sup _{\alpha, \beta}\left|\frac{1}{M^{s+1} N} \sum_{m=1}^{M} \sum_{n=1}^{N} m^{s} X(m, n) e^{i\left[\alpha m+\beta m^{2}\right]}\right| \rightarrow 0$ a.s.. It can be proved along the same line as Kundu and Nandi [7].

Now we choose $L$ large such that $1-L \min \left(\lambda_{11}, \lambda_{21}\right)<0$ and $1-L \min \left(\lambda_{12}, \lambda_{22}\right)<$ 0. Then using again bivariate Taylor series expansion, the 3rd term in equation (25) 
becomes

$$
\begin{aligned}
& \sum_{m=1}^{M} \sum_{n=1}^{N} X(m, n) e^{-i\left(\widetilde{\alpha} m+\widetilde{\beta} m^{2}\right)}=\sum_{m=1}^{M} \sum_{n=1}^{N} X(m, n)\left[e^{-i\left(m \alpha^{0}+m^{2} \beta^{0}\right)}\right. \\
& +\sum_{l=1}^{L-1} \sum_{k=0}^{l} \frac{\left(i\left(\widetilde{\alpha}-\alpha^{0}\right) m\right)^{k}\left(i\left(\widetilde{\beta}-\beta^{0}\right) m^{2}\right)^{l-k}}{k !(l-k) !} e^{-i\left(m \alpha^{0}+m^{2} \beta^{0}\right)} \\
& \left.+\sum_{k=0}^{L} \frac{\left(i\left(\widetilde{\alpha}-\alpha^{0}\right) m\right)^{k}\left(i\left(\widetilde{\beta}-\beta^{0}\right) m^{2}\right)^{L-k}}{k !(L-k) !} e^{-i\left(m \alpha^{*}+m^{2} \beta^{*}\right)}\right] \\
& =O_{p}\left((M N)^{\frac{1}{2}}\right) \\
& +\sum_{l=1}^{L-1} \sum_{k=0}^{l} \frac{1}{k !(l-k) !} O_{p}\left(M^{-k-k \lambda_{11}} N^{-k \lambda_{12}}\right) O_{p}\left(M^{-2(l-k)-(l-k) \lambda_{21}} N^{-(l-k) \lambda_{22}}\right) O_{p}\left(M^{k+2(l-k)+\frac{1}{2}} N^{\frac{1}{2}}\right) \\
& +\sum_{k=0}^{L} \frac{1}{k !(L-k) !} O_{p}\left(M^{-k-k \lambda_{11}} N^{-k \lambda_{12}}\right) O_{p}\left(M^{-2(L-k)-(L-k) \lambda_{21}} N^{-(L-k) \lambda_{22}}\right) O_{p}\left(M^{k+2(L-k)+1} N\right) \\
& =O_{p}\left((M N)^{\frac{1}{2}}\right)+\sum_{l=1}^{L-1} \sum_{k=0}^{l} \frac{1}{k !(l-k) !}\left[O_{p}\left(M^{\frac{1}{2}-k \lambda_{11}-(l-k) \lambda_{21}} N^{\frac{1}{2}-k \lambda_{12}-(l-k) \lambda_{22}}\right)\right] \\
& +\sum_{k=0}^{L} \frac{1}{k !(L-k) !}\left[O_{p}\left(M^{1-k \lambda_{11}-(L-k) \lambda_{21}} N^{1-k \lambda_{12}-(L-k) \lambda_{22}}\right)\right] \\
& =O_{p}\left((M N)^{\frac{1}{2}}\right)+\sum_{l=1}^{L-1} O_{p}\left((M N)^{\frac{1}{2}}\right) \frac{1}{l !}\left(M^{-\lambda_{11}} N^{-\lambda_{12}}+M^{-\lambda_{21}} N^{-\lambda_{22}}\right)^{l} \\
& +O_{p}(M N) \frac{1}{L !}\left(M^{-\lambda_{11}} N^{-\lambda_{12}}+M^{-\lambda_{21}} N^{-\lambda_{22}}\right)^{L} \\
& =O_{p}\left((M N)^{\frac{1}{2}}\right)+\sum_{l=1}^{L-1} O_{p}\left((M N)^{\frac{1}{2}}\right) \frac{1}{l !}\left(M^{-\min \left(\lambda_{11}, \lambda_{21}\right)} N^{-\min \left(\lambda_{12}, \lambda_{22}\right)}\right)^{l} \\
& +O_{p}(M N) \frac{1}{L !}\left(M^{-\min \left(\lambda_{11}, \lambda_{21}\right)} N^{-\min \left(\lambda_{12}, \lambda_{22}\right)}\right)^{L} \\
& =O_{p}\left((M N)^{\frac{1}{2}}\right)+\sum_{l=1}^{L-1} O_{p}\left((M N)^{\frac{1}{2}}\right) \frac{1}{l !}\left(M^{-\min \left(\lambda_{11}, \lambda_{21}\right)} N^{-\min \left(\lambda_{12}, \lambda_{22}\right)}\right)^{l}+O_{p}(1) \\
& =O_{p}\left((M N)^{\frac{1}{2}}\right) \text {. }
\end{aligned}
$$

Then equation (25) becomes

$$
\begin{aligned}
Q_{M N}^{\alpha, \beta} & =\left(\frac{A_{N}^{*}-i B_{N}^{*}}{2}\right) O_{p}(M)\left[1+O_{p}\left(M^{-\min \left(\lambda_{11}, \lambda_{21}\right)} N^{-\min \left(\lambda_{12}, \lambda_{22}\right)}\right)\right] \\
& +\left(\frac{A_{N}^{*}+i B_{N}^{*}}{2}\right) o_{p}(M)+O_{p}\left((M N)^{\frac{1}{2}}\right) \\
& =\left(\frac{A_{N}^{*}-i B_{N}^{*}}{2}\right) O_{p}(M)=\left(\frac{A^{0}+B^{0}}{2}+i \frac{A^{0}-B^{0}}{2}\right) O_{p}(M N) .
\end{aligned}
$$




\section{Lemma 4.}

$$
\begin{aligned}
P_{M N}^{\alpha} & =\sum_{m=1}^{M} \sum_{n=1}^{N} X(m, n)\left(m-\frac{M}{2}\right) e^{-i\left(\alpha^{0} m+\beta^{0} m^{2}\right)} \\
& +\left(\frac{A^{0}+B^{0}}{2}+i \frac{A^{0}-B^{0}}{2}\right) i\left(\alpha^{0}-\widetilde{\alpha}\right) O\left(M^{3} N\right)\left[1+O_{p}\left(M^{-\min \left(\lambda_{11}, \lambda_{21}\right)} N^{-\min \left(\lambda_{12}, \lambda_{22}\right)}\right)\right]
\end{aligned}
$$

Proof

$$
\begin{aligned}
& P_{M N}^{\alpha}=\sum_{m=1}^{M} \sum_{n=1}^{N} y(m, n)\left(m-\frac{M}{2}\right) e^{-i\left(\widetilde{\alpha} m+\widetilde{\beta} m^{2}\right)} \\
& =\sum_{m=1}^{M} \sum_{n=1}^{N} X(m, n)\left(m-\frac{M}{2}\right) e^{-i\left(\widetilde{\alpha} m+\widetilde{\beta} m^{2}\right)}+\sum_{m=1}^{M}\left(\frac{A_{N}^{*}-i B_{N}^{*}}{2}\right)\left(m-\frac{M}{2}\right) e^{i\left[\left(\alpha^{0}-\widetilde{\alpha}\right) m+\left(\beta^{0}-\widetilde{\beta}\right) m^{2}\right]} \\
& \\
& \quad+\sum_{m=1}^{M}\left(\frac{A_{N}^{*}+i B_{N}^{*}}{2}\right)\left(m-\frac{M}{2}\right) e^{-i\left[\left(\alpha^{0}+\widetilde{\alpha}\right) m+\left(\beta^{0}+\widetilde{\beta}\right) m^{2}\right]}
\end{aligned}
$$

Now $\sum_{m=1}^{M}\left(m-\frac{M}{2}\right) e^{i\left[\left(\alpha^{0}-\widetilde{\alpha}\right) m+\left(\beta^{0}-\widetilde{\beta}\right) m^{2}\right]}$

$$
\begin{aligned}
& =\sum_{m=1}^{M}\left(m-\frac{M}{2}\right)\left[e^{i\left[m\left(\alpha^{0}-\alpha^{0}\right)+m^{2}\left(\beta^{0}-\beta^{0}\right)\right]}+i\left(\alpha^{0}-\widetilde{\alpha}\right) m+i\left(\beta^{0}-\widetilde{\beta}\right) m^{2}\right. \\
& \quad+\frac{i^{2}\left(\alpha^{0}-\widetilde{\alpha}\right)^{2} m^{2}}{2 !} e^{i\left[m\left(\alpha^{0}-\alpha^{*}\right)+m^{2}\left(\beta^{0}-\beta^{*}\right)\right]}+\frac{i^{2}\left(\beta^{0}-\widetilde{\beta}\right)^{2} m^{4}}{2 !} e^{i\left[m\left(\alpha^{0}-\alpha^{*}\right)+m^{2}\left(\beta^{0}-\beta^{*}\right)\right]} \\
& \left.\quad+\frac{2 i^{2}\left(\alpha^{0}-\widetilde{\alpha}\right)\left(\beta^{0}-\widetilde{\beta}\right) m^{3}}{2 !} e^{i\left[m\left(\alpha^{0}-\alpha^{*}\right)+m^{2}\left(\beta^{0}-\beta^{*}\right)\right]}\right] \\
& =O(M)+i\left(\alpha^{0}-\widetilde{\alpha}\right) O\left(M^{3}\right)+i\left(\alpha^{0}-\widetilde{\alpha}\right) O_{p}\left(M^{-1-\lambda_{11}} N^{-\lambda_{12}}\right) O_{p}\left(M^{4}\right) \\
& \quad+i\left(\alpha^{0}-\widetilde{\alpha}\right) O_{p}\left(M^{-2-\lambda_{21}} N^{-\lambda_{22}}\right) O_{p}\left(M^{5}\right) \\
& \quad+O_{p}\left(M^{-2-\lambda_{21}} N^{-\lambda_{22}}\right) O\left(M^{4}\right)+O_{p}\left(M^{-4-2 \lambda_{21}} N^{-2 \lambda_{22}}\right) O_{p}\left(M^{6}\right) \\
& =O_{p}(M)+i\left(\alpha^{0}-\widetilde{\alpha}\right) O\left(M^{3}\right)\left[1+O_{p}\left(M^{-\lambda_{11}} N^{-\lambda_{12}}\right)+O_{p}\left(M^{-\lambda_{21}} N^{-\lambda_{22}}\right)\right] \\
& \quad+O_{p}\left(M^{2}\right)\left[O_{p}\left(M^{-\lambda_{21}} N^{-\lambda_{22}}\right)+O_{p}\left(M^{-2 \lambda_{21}} N^{-2 \lambda_{22}}\right)\right] \\
& =o_{p}\left(M^{2}\right)+i\left(\alpha^{0}-\widetilde{\alpha}\right) O\left(M^{3}\right)\left[1+O_{p}\left(M^{-\lambda_{11}} N^{-\lambda_{12}}\right)+O_{p}\left(M^{-\lambda_{21}} N^{-\lambda_{22}}\right)\right]
\end{aligned}
$$




$$
=o_{p}\left(M^{2}\right)+i\left(\alpha^{0}-\widetilde{\alpha}\right) O\left(M^{3}\right)\left[1+O_{p}\left(M^{-\min \left(\lambda_{11}, \lambda_{21}\right)} N^{-\min \left(\lambda_{12}, \lambda_{22}\right)}\right] .\right.
$$

Bivariate Taylor series gives 1st term in equation (26) as

$$
\begin{aligned}
& \sum_{m=1}^{M} \sum_{n=1}^{N} X(m, n)\left(m-\frac{M}{2}\right) e^{-i\left(\widetilde{\alpha} m+\widetilde{\beta} m^{2}\right)}=\sum_{m=1}^{M} \sum_{n=1}^{N} X(m, n)\left(m-\frac{M}{2}\right)\left[e^{-i\left(m \alpha^{0}+m^{2} \beta^{0}\right)}\right. \\
& +\sum_{l=1}^{L-1} \sum_{k=0}^{l} \frac{\left(i\left(\widetilde{\alpha}-\alpha^{0}\right) m\right)^{k}\left(i\left(\widetilde{\beta}-\beta^{0}\right) m^{2}\right)^{l-k}}{k !(l-k) !} e^{-i\left(m \alpha^{0}+m^{2} \beta^{0}\right)} \\
& \left.+\sum_{k=0}^{L} \frac{\left(i\left(\widetilde{\alpha}-\alpha^{0}\right) m\right)^{k}\left(i\left(\widetilde{\beta}-\beta^{0}\right) m^{2}\right)^{L-k}}{k !(L-k) !} e^{-i\left(m \alpha^{*}+m^{2} \beta^{*}\right)}\right] \\
& =\sum_{m=1}^{M} \sum_{n=1}^{N} X(m, n)\left(m-\frac{M}{2}\right) e^{-i\left(m \alpha^{0}+m^{2} \beta^{0}\right)} \\
& +\sum_{l=1}^{L-1} \sum_{k=0}^{l} \frac{1}{k !(l-k) !} O_{p}\left(M^{-k-k \lambda_{11}} N^{-k \lambda_{12}}\right) O_{p}\left(M^{-2(l-k)-(l-k) \lambda_{21}} N^{-(l-k) \lambda_{22}}\right) O_{p}\left(M^{k+2(l-k)+\frac{3}{2}} N^{\frac{1}{2}}\right) \\
& +\sum_{k=0}^{L} \frac{1}{k !(L-k) !} O_{p}\left(M^{-k-k \lambda_{11}} N^{-k \lambda_{12}}\right) O_{p}\left(M^{-2(L-k)-(L-k) \lambda_{21}} N^{-(L-k) \lambda_{22}}\right) O_{p}\left(M^{k+2(L-k)+2} N\right) \\
& =\sum_{m=1}^{M} \sum_{n=1}^{N} X(m, n)\left(m-\frac{M}{2}\right) e^{-i\left(m \alpha^{0}+m^{2} \beta^{0}\right)} \\
& +\sum_{l=1}^{L-1} \sum_{k=0}^{l} \frac{1}{k !(l-k) !}\left[O_{p}\left(M^{\frac{3}{2}-k \lambda_{11}-(l-k) \lambda_{21}} N^{\frac{1}{2}-k \lambda_{12}-(l-k) \lambda_{22}}\right)\right] \\
& +\sum_{k=0}^{L} \frac{1}{k !(L-k) !}\left[O_{p}\left(M^{2-k \lambda_{11}-(L-k) \lambda_{21}} N^{1-k \lambda_{12}-(L-k) \lambda_{22}}\right)\right] \\
& =\sum_{m=1}^{M} \sum_{n=1}^{N} X(m, n)\left(m-\frac{M}{2}\right) e^{-i\left(m \alpha^{0}+m^{2} \beta^{0}\right)}+\sum_{l=1}^{L-1} O_{p}\left(M^{\frac{3}{2}} N^{\frac{1}{2}}\right) \frac{1}{l !}\left(M^{-\lambda_{11}} N^{-\lambda_{12}}+M^{-\lambda_{21}} N^{-\lambda_{22}}\right)^{l} \\
& +O_{p}\left(M^{2} N\right) \frac{1}{L !}\left(M^{-\lambda_{11}} N^{-\lambda_{12}}+M^{-\lambda_{21}} N^{-\lambda_{22}}\right)^{L} \\
& =\sum_{m=1}^{M} \sum_{n=1}^{N} X(m, n)\left(m-\frac{M}{2}\right) e^{-i\left(m \alpha^{0}+m^{2} \beta^{0}\right)}+\sum_{l=1}^{L-1} O_{p}\left(M^{\frac{3}{2}} N^{\frac{1}{2}}\right) \frac{1}{l !}\left(M^{-\min \left(\lambda_{11}, \lambda_{21}\right)} N^{-\min \left(\lambda_{12}, \lambda_{22}\right)}\right)^{l} \\
& +O_{p}\left(M^{2} N\right) \frac{1}{L !}\left(M^{-\min \left(\lambda_{11}, \lambda_{21}\right)} N^{-\min \left(\lambda_{12}, \lambda_{22}\right)}\right)^{L} \\
& =\sum_{m=1}^{M} \sum_{n=1}^{N} X(m, n)\left(m-\frac{M}{2}\right) e^{-i\left(m \alpha^{0}+m^{2} \beta^{0}\right)} \\
& +\sum_{l=1}^{L-1} O_{p}\left(M^{\frac{3}{2}} N^{\frac{1}{2}}\right) \frac{1}{l !}\left(M^{-\min \left(\lambda_{11}, \lambda_{21}\right)} N^{-\min \left(\lambda_{12}, \lambda_{22}\right)}\right)^{l}+O_{p}(M) \\
& =\sum_{m=1}^{M} \sum_{n=1}^{N} X(m, n)\left(m-\frac{M}{2}\right) e^{-i\left(m \alpha^{0}+m^{2} \beta^{0}\right)}+o_{p}\left(M^{\frac{3}{2}} N^{\frac{1}{2}}\right) \text {. }
\end{aligned}
$$


For 3rd term in equation (26) we calculate

$$
\sum_{m=1}^{M}\left(m-\frac{M}{2}\right) e^{-i\left[\left(\alpha^{0}+\widetilde{\alpha}\right) m+\left(\beta^{0}+\widetilde{\beta}\right) m^{2}\right]}=\sum_{m=1}^{M}\left(m-\frac{M}{2}\right) O_{p}(1)=O_{p}(M) .
$$

Then equation (26) becomes

$$
\begin{aligned}
P_{M N}^{\alpha}= & \left(\frac{A_{N}^{*}-i B_{N}^{*}}{2}\right) i\left(\alpha^{0}-\widetilde{\alpha}\right) O\left(M^{3}\right)\left[1+O_{p}\left(M^{-\min \left(\lambda_{11}, \lambda_{21}\right)} N^{-\min \left(\lambda_{12}, \lambda_{22}\right)}\right]+o_{p}\left(M^{2}\right)\right. \\
+ & \left(\frac{A_{N}^{*}+i B_{N}^{*}}{2}\right) O_{p}(M)+o_{p}\left(M^{\frac{3}{2}} N^{\frac{1}{2}}\right)+\sum_{m=1}^{M} \sum_{n=1}^{N} X(m, n)\left(m-\frac{M}{2}\right) e^{-i\left(\alpha^{0} m+\beta^{0} m^{2}\right)} \\
= & \sum_{m=1}^{M} \sum_{n=1}^{N} X(m, n)\left(m-\frac{M}{2}\right) e^{-i\left(\alpha^{0} m+\beta^{0} m^{2}\right)} \\
& +\left(\frac{A_{N}^{*}-i B_{N}^{*}}{2}\right) i\left(\alpha^{0}-\widetilde{\alpha}\right) O\left(M^{3}\right)\left[1+O_{p}\left(M^{-\min \left(\lambda_{11}, \lambda_{21}\right)} N^{-\min \left(\lambda_{12}, \lambda_{22}\right)}\right)\right] \\
= & \sum_{m=1}^{M} \sum_{n=1}^{N} X(m, n)\left(m-\frac{M}{2}\right) e^{-i\left(\alpha^{0} m+\beta^{0} m^{2}\right)} \\
& +\left(\frac{A^{0}+B^{0}}{2}+i \frac{A^{0}-B^{0}}{2}\right) i\left(\alpha^{0}-\widetilde{\alpha}\right) O\left(M^{3} N\right)\left[1+O_{p}\left(M^{-\min \left(\lambda_{11}, \lambda_{21}\right)} N^{-\min \left(\lambda_{12}, \lambda_{22}\right)}\right)\right] .
\end{aligned}
$$

\section{Lemma 5.}

$$
\begin{aligned}
P_{M N}^{\beta} & =\sum_{m=1}^{M} \sum_{n=1}^{N} X(m, n)\left(m^{2}-\frac{M^{2}}{3}\right) e^{-i\left(\beta^{0} m+\beta^{0} m^{2}\right)} \\
& +\left(\frac{A^{0}+B^{0}}{2}+i \frac{A^{0}-B^{0}}{2}\right) i\left(\beta^{0}-\widetilde{\beta}\right) O\left(M^{5} N\right)\left[1+O_{p}\left(M^{-\min \left(\lambda_{11}, \lambda_{21}\right)} N^{-\min \left(\lambda_{12}, \lambda_{22}\right)}\right)\right]
\end{aligned}
$$

\section{Proof}

$$
\begin{aligned}
P_{M N}^{\beta}= & \sum_{m=1}^{M} \sum_{n=1}^{N} y(m, n)\left(m^{2}-\frac{M^{2}}{3}\right) e^{-i\left(\widetilde{\alpha} m+\widetilde{\beta} m^{2}\right)} \\
= & \sum_{m=1}^{M} \sum_{n=1}^{N} X(m, n)\left(m^{2}-\frac{M^{2}}{3}\right) e^{-i\left(\widetilde{\alpha} m+\widetilde{\beta} m^{2}\right)}+\sum_{m=1}^{M}\left(\frac{A_{N}^{*}-i B_{N}^{*}}{2}\right)\left(m^{2}-\frac{M^{2}}{3}\right) e^{i\left[\left(\alpha^{0}-\widetilde{\alpha}\right) m+\left(\beta^{0}-\widetilde{\beta}\right) m^{2}\right]} \\
& +\sum_{m=1}^{M}\left(\frac{A_{N}^{*}+i B_{N}^{*}}{2}\right)\left(m-\frac{M}{2}\right) e^{-i\left[\left(\alpha^{0}+\widetilde{\alpha}\right) m+\left(\beta^{0}+\widetilde{\beta}\right) m^{2}\right]}
\end{aligned}
$$

To get 3rd term in equation (27) we calculate

$$
\sum_{m=1}^{M}\left(m^{2}-\frac{M^{2}}{3}\right) e^{-i\left[\left(\alpha^{0}+\widetilde{\alpha}\right) m+\left(\beta^{0}+\widetilde{\beta}\right) m^{2}\right]}=\sum_{m=1}^{M}\left(m^{2}-\frac{M^{2}}{3}\right) O_{p}(1)=O_{p}\left(M^{2}\right) .
$$


Expanding using bivariate Taylor series 1st term in equation (27) becomes

$$
\begin{aligned}
& \sum_{m=1}^{M} \sum_{n=1}^{N} X(m, n)\left(m^{2}-\frac{M^{2}}{3}\right) e^{-i\left(\widetilde{\alpha} m+\widetilde{\beta} m^{2}\right)}=\sum_{m=1}^{M} \sum_{n=1}^{N} X(m, n)\left(m^{2}-\frac{M^{2}}{3}\right)\left[e^{-i\left(m \alpha^{0}+m^{2} \beta^{0}\right)}\right. \\
& +\sum_{l=1}^{L-1} \sum_{k=0}^{l} \frac{\left(i\left(\widetilde{\alpha}-\alpha^{0}\right) m\right)^{k}\left(i\left(\widetilde{\beta}-\beta^{0}\right) m^{2}\right)^{l-k}}{k !(l-k) !} e^{-i\left(m \alpha^{0}+m^{2} \beta^{0}\right)} \\
& \left.+\sum_{k=0}^{L} \frac{\left(i\left(\widetilde{\alpha}-\alpha^{0}\right) m\right)^{k}\left(i\left(\widetilde{\beta}-\beta^{0}\right) m^{2}\right)^{L-k}}{k !(L-k) !} e^{-i\left(m \alpha^{*}+m^{2} \beta^{*}\right)}\right] \\
& =\sum_{m=1}^{M} \sum_{n=1}^{N} X(m, n)\left(m^{2}-\frac{M^{2}}{3}\right) e^{-i\left(m \alpha^{0}+m^{2} \beta^{0}\right)} \\
& +\sum_{l=1}^{L-1} \sum_{k=0}^{l} \frac{1}{k !(l-k) !} O_{p}\left(M^{-k-k \lambda_{11}} N^{-k \lambda_{12}}\right) O_{p}\left(M^{-2(l-k)-(l-k) \lambda_{21}} N^{-(l-k) \lambda_{22}}\right) O_{p}\left(M^{k+2(l-k)+\frac{5}{2}} N^{\frac{1}{2}}\right) \\
& +\sum_{k=0}^{L} \frac{1}{k !(L-k) !} O_{p}\left(M^{-k-k \lambda_{11}} N^{-k \lambda_{12}}\right) O_{p}\left(M^{-2(L-k)-(L-k) \lambda_{21}} N^{-(L-k) \lambda_{22}}\right) O_{p}\left(M^{k+2(L-k)+3} N\right) \\
& =\sum_{m=1}^{M} \sum_{n=1}^{N} X(m, n)\left(m^{2}-\frac{M^{2}}{3}\right) e^{-i\left(m \alpha^{0}+m^{2} \beta^{0}\right)} \\
& +\sum_{l=1}^{L-1} \sum_{k=0}^{l} \frac{1}{k !(l-k) !}\left[O_{p}\left(M^{\frac{5}{2}-k \lambda_{11}-(l-k) \lambda_{21}} N^{\frac{1}{2}-k \lambda_{12}-(l-k) \lambda_{22}}\right)\right] \\
& +\sum_{k=0}^{L} \frac{1}{k !(L-k) !}\left[O_{p}\left(M^{3-k \lambda_{11}-(L-k) \lambda_{21}} N^{1-k \lambda_{12}-(L-k) \lambda_{22}}\right)\right] \\
& =\sum_{m=1}^{M} \sum_{n=1}^{N} X(m, n)\left(m^{2}-\frac{M^{2}}{3}\right) e^{-i\left(m \alpha^{0}+m^{2} \beta^{0}\right)}+\sum_{l=1}^{L-1} O_{p}\left(M^{\frac{5}{2}} N^{\frac{1}{2}}\right) \frac{1}{l !}\left(M^{-\lambda_{11}} N^{-\lambda_{12}}+M^{-\lambda_{21}} N^{-\lambda_{22}}\right)^{l} \\
& +O_{p}\left(M^{3} N\right) \frac{1}{L !}\left(M^{-\lambda_{11}} N^{-\lambda_{12}}+M^{-\lambda_{21}} N^{-\lambda_{22}}\right)^{L} \\
& =\sum_{m=1}^{M} \sum_{n=1}^{N} X(m, n)\left(m^{2}-\frac{M^{2}}{3}\right) e^{-i\left(m \alpha^{0}+m^{2} \beta^{0}\right)}+\sum_{l=1}^{L-1} O_{p}\left(M^{\frac{5}{2}} N^{\frac{1}{2}}\right) \frac{1}{l !}\left(M^{-\min \left(\lambda_{11}, \lambda_{21}\right)} N^{-\min \left(\lambda_{12}, \lambda_{22}\right)}\right)^{l} \\
& +O_{p}\left(M^{3} N\right) \frac{1}{L !}\left(M^{-\min \left(\lambda_{11}, \lambda_{21}\right)} N^{-\min \left(\lambda_{12}, \lambda_{22}\right)}\right)^{L} \\
& =\sum_{m=1}^{M} \sum_{n=1}^{N} X(m, n)\left(m^{2}-\frac{M^{2}}{3}\right) e^{-i\left(m \alpha^{0}+m^{2} \beta^{0}\right)} \\
& +\sum_{l=1}^{L-1} O_{p}\left(M^{\frac{5}{2}} N^{\frac{1}{2}}\right) \frac{1}{l !}\left(M^{-\min \left(\lambda_{11}, \lambda_{21}\right)} N^{-\min \left(\lambda_{12}, \lambda_{22}\right)}\right)^{l}+O_{p}\left(M^{2}\right) \\
& =\sum_{m=1}^{M} \sum_{n=1}^{N} X(m, n)\left(m^{2}-\frac{M^{2}}{3}\right) e^{-i\left(m \alpha^{0}+m^{2} \beta^{0}\right)}+o_{p}\left(M^{\frac{5}{2}} N^{\frac{1}{2}}\right) .
\end{aligned}
$$


Taylor series expansion gives 2nd term in equation (27) as

$$
\begin{aligned}
\sum_{m=1}^{M}( & \left.m^{2}-\frac{M^{2}}{3}\right) e^{i\left[\left(\alpha^{0}-\widetilde{\alpha}\right) m+\left(\beta^{0}-\widetilde{\beta}\right) m^{2}\right]} \\
=\sum_{m=1}^{M} & \left(m^{2}-\frac{M^{2}}{3}\right)\left[e^{i\left[m\left(\alpha^{0}-\alpha^{0}\right)+m^{2}\left(\beta^{0}-\beta^{0}\right)\right]}+i\left(\alpha^{0}-\widetilde{\alpha}\right) m+i\left(\beta^{0}-\widetilde{\beta}\right) m^{2}\right. \\
& +\frac{i^{2}\left(\alpha^{0}-\widetilde{\alpha}\right)^{2} m^{2}}{2 !} e^{i\left[m\left(\alpha^{0}-\alpha^{*}\right)+m^{2}\left(\beta^{0}-\beta^{*}\right)\right]}+\frac{i^{2}\left(\beta^{0}-\widetilde{\beta}\right)^{2} m^{4}}{2 !} e^{i\left[m\left(\alpha^{0}-\alpha^{*}\right)+m^{2}\left(\beta^{0}-\beta^{*}\right)\right]} \\
& \left.+\frac{2 i^{2}\left(\alpha^{0}-\widetilde{\alpha}\right)\left(\beta^{0}-\widetilde{\beta}\right) m^{3}}{2 !} e^{i\left[m\left(\alpha^{0}-\alpha^{*}\right)+m^{2}\left(\beta^{0}-\beta^{*}\right)\right]}\right] \\
= & \left(M^{2}\right)+i\left(\beta^{0}-\widetilde{\beta}\right) O\left(M^{5}\right)+i\left(\beta^{0}-\widetilde{\beta}\right) O_{p}\left(M^{-1-\lambda_{11}} N^{-\lambda_{12}}\right) O_{p}\left(M^{6}\right) \\
& +i\left(\beta^{0}-\widetilde{\beta}\right) O_{p}\left(M^{-2-\lambda_{21}} N^{-\lambda_{22}}\right) O_{p}\left(M^{7}\right) \\
& +O_{p}\left(M^{-1-\lambda_{11}} N^{-\lambda_{12}}\right) O\left(M^{4}\right)+O_{p}\left(M^{-2-2 \lambda_{11}} N^{-2 \lambda_{12}}\right) O_{p}\left(M^{5}\right) \\
= & O_{p}\left(M^{2}\right)+i\left(\beta^{0}-\widetilde{\beta}\right) O\left(M^{5}\right)\left[1+O_{p}\left(M^{-\lambda_{11}} N^{-\lambda_{12}}\right)+O_{p}\left(M^{-\lambda_{21}} N^{-\lambda_{22}}\right)\right] \\
& +O_{p}\left(M^{3}\right)\left[O_{p}\left(M^{-\lambda_{11}} N^{-\lambda_{12}}\right)+O_{p}\left(M^{-2 \lambda_{11}} N^{-2 \lambda_{12}}\right)\right] \\
= & o_{p}\left(M^{3}\right)+i\left(\beta^{0}-\widetilde{\beta}\right) O\left(M^{5}\right)\left[1+O_{p}\left(M^{-\lambda_{11}} N^{-\lambda_{12}}\right)+O_{p}\left(M^{-\lambda_{21}} N^{-\lambda_{22}}\right)\right] \\
= & o_{p}\left(M^{3}\right)+i\left(\beta^{0}-\widetilde{\beta}\right) O\left(M^{5}\right)\left[1+O_{p}\left(M^{-\min \left(\lambda_{11}, \lambda_{21}\right)} N^{-\min \left(\lambda_{12}, \lambda_{22}\right)}\right] .\right.
\end{aligned}
$$

Then equation (27) becomes

$$
\begin{aligned}
P_{M N}^{\beta}= & \left(\frac{A_{N}^{*}-i B_{N}^{*}}{2}\right) i\left(\beta^{0}-\widetilde{\beta}\right) O\left(M^{5}\right)\left[1+O_{p}\left(M^{-\min \left(\lambda_{11}, \lambda_{21}\right)} N^{-\min \left(\lambda_{12}, \lambda_{22}\right)}\right]+o_{p}\left(M^{3}\right)\right. \\
+ & \left(\frac{A_{N}^{*}+i B_{N}^{*}}{2}\right) O_{p}\left(M^{2}\right)+o_{p}\left(M^{\frac{5}{2}} N^{\frac{1}{2}}\right)+\sum_{m=1}^{M} \sum_{n=1}^{N} X(m, n)\left(m^{2}-\frac{M^{2}}{3}\right) e^{-i\left(\beta^{0} m+\beta^{0} m^{2}\right)} \\
= & \sum_{m=1}^{M} \sum_{n=1}^{N} X(m, n)\left(m^{2}-\frac{M^{2}}{3}\right) e^{-i\left(\beta^{0} m+\beta^{0} m^{2}\right)} \\
& +\left(\frac{A_{N}^{*}-i B_{N}^{*}}{2}\right) i\left(\beta^{0}-\widetilde{\beta}\right) O\left(M^{5}\right)\left[1+O_{p}\left(M^{-\min \left(\lambda_{11}, \lambda_{21}\right)} N^{-\min \left(\lambda_{12}, \lambda_{22}\right)}\right)\right] \\
= & \sum_{m=1}^{M} \sum_{n=1}^{N} X(m, n)\left(m^{2}-\frac{M^{2}}{3}\right) e^{-i\left(\beta^{0} m+\beta^{0} m^{2}\right)} \\
& +\left(\frac{A^{0}+B^{0}}{2}+i \frac{A^{0}-B^{0}}{2}\right) i\left(\beta^{0}-\widetilde{\beta}\right) O\left(M^{5} N\right)\left[1+O_{p}\left(M^{-\min \left(\lambda_{11}, \lambda_{21}\right)} N^{-\min \left(\lambda_{12}, \lambda_{22}\right)}\right)\right] .
\end{aligned}
$$


Proof of Theorem 1: Note that

$$
\sum_{n=1}^{N} y(m, n)=A_{N}^{*} \cos \left(\alpha^{0} m+\beta^{0} m^{2}\right)+B_{N}^{*} \sin \left(\alpha^{0} m+\beta^{0} m^{2}\right)+\sum_{n=1}^{N} X(m, n)
$$

where $A_{N}^{*}=\sum_{n=1}^{N}\left[A^{0} \cos \left(\gamma^{0} n+\delta^{0} n^{2}\right)+B^{0} \sin \left(\gamma^{0} n+\delta^{0} n^{2}\right)\right]=\left(A^{0}+B^{0}\right) O(N)$ and $B_{N}^{*}=\sum_{n=1}^{N}\left[B^{0} \cos \left(\gamma^{0} n+\delta^{0} n^{2}\right)-A^{0} \sin \left(\gamma^{0} n+\delta^{0} n^{2}\right)\right]=\left(B^{0}-A^{0}\right) O(N)$.

$Q_{M N}^{\alpha, \beta}=\left(\frac{A^{0}+B^{0}}{2}+i \frac{A^{0}-B^{0}}{2}\right) O_{p}(M N)$ using Lemma 3

and using Lemma 4

$$
\begin{aligned}
P_{M N}^{\alpha} & =\sum_{m=1}^{M} \sum_{n=1}^{N} X(m, n)\left(m-\frac{M}{2}\right) e^{-i\left(\alpha^{0} m+\beta^{0} m^{2}\right)} \\
& +\left(\frac{A^{0}+B^{0}}{2}+i \frac{A^{0}-B^{0}}{2}\right) i\left(\alpha^{0}-\widetilde{\alpha}\right) O\left(M^{3} N\right)\left[1+O_{p}\left(M^{-\min \left(\lambda_{11}, \lambda_{21}\right)} N^{-\min \left(\lambda_{12}, \lambda_{22}\right)}\right)\right]
\end{aligned}
$$

then $\quad \widetilde{\widetilde{\alpha}}=\widetilde{\alpha}+\frac{48}{M^{2}} \operatorname{Im}\left[\frac{P_{M N}^{\alpha}}{Q_{M N}^{\alpha, \beta}}\right]$

$$
\left.\begin{array}{c}
\widetilde{\widetilde{\alpha}}=\widetilde{\alpha}+\frac{48}{M^{2}} \operatorname{Im}\left[\frac{\left(\frac{A_{N}^{*}-i B_{N}^{*}}{2}\right) i\left(\alpha^{0}-\widetilde{\alpha}\right) O\left(M^{3}\right)\left[1+O_{p}\left(M^{-\min \left(\lambda_{11}, \lambda_{21}\right)} N^{-\min \left(\lambda_{12}, \lambda_{22}\right)}\right]\right.}{O_{p}(M)\left(\frac{A_{N}^{*}-i B_{N}^{*}}{2}\right)}\right. \\
\left.+\frac{\sum_{m=1}^{M} \sum_{n=1}^{N} X(m, n)\left(m-\frac{M}{2}\right) e^{-i\left(\alpha^{0} m+\beta^{0} m^{2}\right)}}{O_{p}(M)\left(\frac{A_{N}^{*}-i B_{N}^{*}}{2}\right)}\right] \\
\widetilde{\widetilde{\alpha}}=\alpha^{0}+\left(\alpha^{0}-\widetilde{\alpha}\right) O_{p}\left(M^{-\min \left(\lambda_{11}, \lambda_{21}\right)} N^{-\min \left(\lambda_{12}, \lambda_{22}\right)}\right.
\end{array}\right]
$$$$
+\frac{48}{M^{2}} \operatorname{Im}\left[\frac{\sum_{m=1}^{M} \sum_{n=1}^{N} X(m, n)\left(m-\frac{M}{2}\right) e^{-i\left(\alpha^{0} m+\beta^{0} m^{2}\right)}}{\left(\frac{A^{0}+B^{0}}{2}+i \frac{A^{0}-B^{0}}{2}\right) O_{p}(M N)}\right] \text {. }
$$

Now the variance of $\frac{48}{M^{-3 / 2} N^{-1 / 2}} \operatorname{Im}\left[\frac{\sum_{m=1}^{M} \sum_{n=1}^{N} X(m, n)\left(m-\frac{M}{2}\right) e^{-i\left(\alpha^{0} m+\beta^{0} m^{2}\right)}}{\left(\frac{A^{0}+B^{0}}{2}+i \frac{A^{0}-B^{0}}{2}\right)}\right]$ using

Lemma 1 and 2, is asymptotically same as variance of LSE of $\alpha^{0}$. So $\widetilde{\widetilde{\alpha}}-\alpha^{0}=$ $O_{p}\left(M^{\left(-1-\lambda_{11}\right)} N^{\left(-\lambda_{12}\right)}\right)$ where $\lambda_{11} \in\left(0, \frac{1}{2}\right)$ then $\widetilde{\widetilde{\alpha}}-\alpha^{0}=O_{p}\left(M^{\left(-1-2 \lambda_{11}\right)} N^{\left(-\lambda_{12}\right)}\right)$ if $\lambda_{11} \leq \frac{1}{4} M^{\frac{3}{2}}\left(\widetilde{\widetilde{\alpha}}-\alpha^{0}\right) \rightarrow N\left(0, \sigma_{1}^{2}\right)$ if $\lambda_{11}>\frac{1}{4}$ by Central Limit Theorem (CLT) of stochastic process in Fuller [3] where $\sigma_{1}^{2}=\frac{384 \sigma^{2} c}{A^{0^{2}}+B^{0^{2}}}$ is variance of LSE of $\alpha^{0}$. 
Proof of TheOrem 2:

$$
\begin{aligned}
P_{M N}^{\beta} & =\sum_{m=1}^{M} \sum_{n=1}^{N} X(m, n)\left(m^{2}-\frac{M^{2}}{3}\right) e^{-i\left(\beta^{0} m+\beta^{0} m^{2}\right)} \\
& +\left(\frac{A^{0}+B^{0}}{2}+i \frac{A^{0}-B^{0}}{2}\right) i\left(\beta^{0}-\widetilde{\beta}\right) O\left(M^{5} N\right)\left[1+O_{p}\left(M^{-\min \left(\lambda_{11}, \lambda_{21}\right)} N^{-\min \left(\lambda_{12}, \lambda_{22}\right)}\right)\right]
\end{aligned}
$$

using Lemma 5 and then $\widetilde{\widetilde{\beta}}=\widetilde{\beta}+\frac{45}{M^{4}} \operatorname{Im}\left[\frac{P_{M N}^{\beta}}{Q_{M N}^{\alpha, \beta}}\right]$

$$
\begin{array}{r}
\widetilde{\beta}=\widetilde{\beta}+\frac{45}{M^{4}} \operatorname{Im}\left[\frac{\left(\frac{A_{N}^{*}-i B_{N}^{*}}{2}\right) i\left(\beta^{0}-\widetilde{\beta}\right) O\left(M^{5}\right)\left[1+O_{p}\left(M^{-\min \left(\lambda_{11}, \lambda_{21}\right)} N^{-\min \left(\lambda_{12}, \lambda_{22}\right)}\right]\right.}{O_{p}(M)\left(\frac{A_{N}^{*}-i B_{N}^{*}}{2}\right)}\right. \\
\left.+\frac{\sum_{m=1}^{M} \sum_{n=1}^{N} X(m, n)\left(m^{2}-\frac{M^{2}}{3}\right) e^{-i\left(\alpha^{0} m+\beta^{0} m^{2}\right)}}{O_{p}(M)\left(\frac{A_{N}^{*}-i B_{N}^{*}}{2}\right)}\right]
\end{array}
$$

$\widetilde{\widetilde{\beta}}=\beta^{0}+\left(\beta^{0}-\widetilde{\beta}\right) O_{p}\left(M^{-\min \left(\lambda_{11}, \lambda_{21}\right)} N^{-\min \left(\lambda_{12}, \lambda_{22}\right)}\right.$

$$
+\frac{45}{M^{4}} \operatorname{Im}\left[\frac{\sum_{m=1}^{M} \sum_{n=1}^{N} X(m, n)\left(m^{2}-\frac{M^{2}}{3}\right) e^{-i\left(\alpha^{0} m+\beta^{0} m^{2}\right)}}{\left(\frac{A^{0}+B^{0}}{2}+i \frac{A^{0}-B^{0}}{2}\right) O_{p}(M N)}\right] .
$$

Now variance of $\frac{45}{M^{-3 / 2} N^{-1 / 2}} \operatorname{Im}\left[\frac{\sum_{m=1}^{M} \sum_{n=1}^{N} X(m, n)\left(m^{2}-\frac{M^{2}}{3}\right) e^{-i\left(\alpha^{0} m+\beta^{0} m^{2}\right)}}{\left(\frac{A^{0}+B^{0}}{2}+i \frac{A^{0}-B^{0}}{2}\right)}\right]$ using

Lemma 1 and 2 , is asymptotically same as variance of LSE of $\beta^{0}$. So $\widetilde{\beta}-\beta^{0}=$ $O_{p}\left(M^{\left(-2-\lambda_{21}\right)}\right)$ where $\lambda_{21} \in\left(0, \frac{1}{2}\right)$ then $\widetilde{\widetilde{\beta}}-\beta^{0}=O_{p}\left(M^{\left(-2-2 \lambda_{21}\right)}\right)$ if $\lambda_{21} \leq \frac{1}{4}$ and $M^{\frac{5}{2}}\left(\widetilde{\widetilde{\beta}}-\beta^{0}\right) \rightarrow N\left(0, \sigma_{2}^{2}\right)$ if $\lambda_{21}>\frac{1}{4}$ by CLT of stochastic processes in Fuller [3] where $\sigma_{2}^{2}=\frac{360 \sigma^{2} c}{A^{0^{2}+B^{0}}}$ is the variance of the LSE of $\beta^{0}$.

Proof of Theorem 3 And 4: Theorems 3 and 4 can be proved along the same line as that of Theorem 1 and 2 by interchanging the role of $m$ and $n$. 


\section{References}

[1] Bai, Z.D., Rao, C.R., Chow, M., Kundu, D. (2003), "Efficient algorithm for estimating the parameters of superimposed complex exponential model", Journal of Statistical Planning and Inference, vol. 110, 23-34.

[2] Cao, F.,Wang, S., Wang, F. (2006), "Cross-Spectral Method Based on 2-D Cross Polynomial Transform for 2-D Chirp Signal Parameter Estimation" ICSP2006 Proceedings, DOI:10.1109/ICOSP.2006.344475.

[3] Fuller, W. (1996), Introduction to statistical time series, 2-nd edition, John Wiley and Sons, New York.

[4] Francos, J.M., Friedlander, B. (1998), "Two-Dimensional Polynomial Phase Signals: Parameter Estimation and Bounds" Multidimensional Systems and Signal Processing, 9, 173-205.

[5] Francos, J.M., Friedlander, B. (1999), "Parameter Estimation of 2-D Random Amplitude Polynomial-Phase Signals", IEEE Transactions on Signal Processing, $47,1795-1810$.

[6] Friedlander, B.,Francos, J.M. (1996), "An Estimation Algorithm for 2-D Polynomial Phase Signals", IEEE Transactions on Image Processing, 5, 1084-1087.

[7] Kundu, D. and Nandi, S. (2008), "Parameter estimation of chirp signals in presence of stationary noise", Statistica Sinica, vol. 18, 187 - 202.

[8] Lahiri, A., Kundu, D. and Mitra, A. (2011), "On parameter estimation of two dimensional chirp signals", submitted for publication.

[9] Peleg, S., Porat, B. (1991), "Estimation and classification of polynomial phase signals" IEEE Transactions on Information Theory, 37, 422-430. 
[10] Rice, J.A. and Rosenblatt, M. (1988), "On frequency estimation", Biometrika, vol. 75,477 - 484 .

[11] Richards, F.S.G. (1961), "A method of maximum likelihood estimation", Journal of the Royal Statistical Society, Ser. B, vol. 23, 469 - 475.

[12] Vinogradov, I.M. (1954), "The method of trigonometrical sums in the theory of numbers" , Interscience, Translated from Russian. Revised and annotated by K. F. Roth and Anne Davenport. Reprint of the 1954 translation. Dover Publications, Inc., Mineola, NY, 2004.

[13] Zhang, H., Liu, Q. (2006) "Estimation of instantaneous frequency rate for multicomponent polynomial phase signals", ICSP2006 Proceedings, 498- 502, DOI: 10.1109/ICOSP.2006.344448.

[14] Zhang, K., Wang, S., Cao, F. (2008), "Product Cubic Phase Function Algorithm for Estimating the Instantaneous Frequency Rate of Multicomponent Twodimensional Chirp Signals", 2008 Congress on Image and Signal Processing, DOI: 10.1109/CISP.2008.352. 